\title{
9. Davidson Taylor \\ Bericht über eine Reise nach Berlin, 20. Juli 1945
}

1. Am Dienstag, dem 17. Juli, erstatteten Mr. Conant, Oberstleutnant Murphy, Dr. Van Eerden ${ }^{61}$ und ich General McClure in Berlin Bericht.

2. Die meisten Teile der Stadt sehen, wenn man sich Tempelhof auf dem Luftweg nähert, wie Ameisenhaufen aus. Die Menschen bewegen sich in langsamen Reihen zwischen den Trümmern, die von der Straße zur Seite geräumt worden sind. Aus der Luft erscheint die Stadt schlimmer zerstört, als dies in Wirklichkeit der Fall ist.

3. Das Flugfeld in Tempelhof befindet sich in ausgezeichnetem Zustand. Zahlreiche Gebäude werden wieder instand gesetzt.

4. Die Quartiermöglichkeiten für USGCC-Personal sind noch immer begrenzt. Was das Casino betrifft, bleibt noch manches zu wünschen übrig. Jedenfalls ist Oberstleutnant Fried ${ }^{61 a}$ dabei, seinen Bereich schnell zu organisieren, und kann sich dann um jeden kümmern, der nach Berlin einreisen darf.

5. General McClure hat sein Quartier in der Nähe des Wannsees. ${ }^{62}$ Die Wachen, die in der Nähe einquartiert sind, haben sich in den Besitz sämtlicher Motorboote an ihrem Ende des Sees gebracht, und irgendwie ist es ihnen gelungen, die Motoren zu reparieren. Wenn sie keinen Dienst haben, rasen sie mit deutschen Mädchen im Boot den See rauf und runter und lassen es sich wohl sein.

6. Die Berliner scheinen stärker erschöpft zu sein als die Londoner nach den VWaffen-Angriffen. Trotzdem ist für sie der Krieg offenbar endgültiger beendet als für die Bevölkerung jeder anderen europäischen Stadt, die ich gesehen habe. Mit dem Krieg brauchen sich die Berliner überhaupt nicht mehr zu beschäftigen. Sie brauchen sich noch nicht einmal den Kopf darüber zu zerbrechen, wie sie sich regieren sollen. Drei Nationen haben das für sie übernommen.

7. Die Franzosen sind in der Stadt nicht als Mitglieder des Viermächte-KontrollRats; ${ }^{63}$ sie unterhalten hier lediglich Verbindungsoffiziere, und diese Offiziere sind

${ }^{61}$ Luther Conant war Chef der ICD Press Control Branch, Adrian Murphy und Dr. Albert Van Eerden gehörten ebenfalls dem ICD an, Murphy als Deputy Director, Van Eerden als Verwaltungsbeamter.

61a Raymond Fried war Commanding Officer bei USGCC ICD.

${ }^{62}$ Wie aus einer handschriftlichen Bemerkung auf dem Originaldokument hervorgeht, bewohnte Robert A. McClure von 1945 bis 1946 das von der amerikanischen Besatzungsmacht beschlagnahmte Haus Heinz Rühmanns.

${ }^{63}$ Der Alliierte Kontrollrat, gebildet auf Grund eines Abkommens vom 14. November 1944 und bestehend aus den Oberbefehlshabern der Streitkräfte der Besatzungsmächte, konstituierte sich erst am 30. Juli 1945. Auf seiner konstituierenden Sitzung billigte er das Abkommen über die Abtrennung einer Besatzungszone (Sektor) innerhalb Groß-Berlins an Frankreich. Seit Anfang Juli befand sich ein französisches Detachement in Berlin, das auch in der Interalliierten Kommandatura mitarbeitete, obwohl der französische Sektor erst am 12. August 1945 gebildet wurde. 
dem Stab der USGCC zugeordnet. Die Franzosen sind auf Betreiben der russischen Regierung von der aktiven Teilnahme ausgeschlossen.

8. Am Morgen des 18. Juli konferierten wir mit Oberst Leonards ${ }^{64}$ „Information Control"-Stab für Berlin und besprachen einige Probleme, die möglicherweise bei dem Treffen der Kommandatura auftauchen könnten. Dieses Treffen war ursprünglich für Dienstag, den 17. Juli, geplant und wurde dann auf Freitag, den 20. Juli, verschoben. Es gab folgende Probleme in den Bereichen Film, Theater und Musik:

a) Sojusintorgkino, die offizielle russische Verleih-Agentur, hat sämtliche UfATheater in Berlin, von denen es 22 geben soll, requiriert. Davon befinden sich fünf oder sechs im amerikanischen Sektor. Darüber hinaus hat Sojusintorgkino einen Vertrag mit einer unbestimmten Zahl anderer Theater abgeschlossen und sich den exklusiven Verleih gesichert. Der Vertrag, der über fünf Jahre läuft, legt fest, daß für jeden deutschen zwei russische Filme gezeigt werden. Maßnahme: Die KontrollOffiziere für Film, Theater und Musik in Berlin werden angewiesen, den russischen Film-Kontroll-Offizier darüber zu informieren, daß der Verleih und die Vorkehrungen für die Vorführungen in der amerikanischen Zone gemäß den Richtlinien der Information Control gehandhabt werden. ${ }^{65}$

b) Die Russen wollen in ihrem Sektor die Oper wiedereröffnen und planen, das Staatsopern-Ensemble für den Admiralspalast zu verpflichten. Als erste Inszenierung ist Glucks „Orpheus“ geplant. Die Russen sind auch daran interessiert, die Eröffnung des Deutschen Opernhauses im britischen Sektor zu ermöglichen. Kostüme für diese beiden Ensembles sind in Eisenach in der amerikanischen Zone gelagert. Maßnahme: Ein amerikanischer und ein britischer Offizier werden sich nach Eisenach begeben, die Kostüme zusammensuchen und sie zurück nach Berlin bringen. ${ }^{66}$

c) Die Berliner Philharmoniker haben Konzerte im russischen Sektor gegeben; die meisten Mitglieder des Orchesters wohnen in Zehlendorf im amerikanischen Sektor. Die Philharmoniker verfügen über keinen ständigen Konzertsaal, und die Russen sind der Meinung, daß die Amerikaner dafür sorgen sollten, daß ein ständiger Ort gefunden wird, wo das Orchester proben kann, und daß wir dabei behilflich sein

${ }^{64} \mathrm{Zu}$ Leonard vgl. Anm. 46a.

${ }^{65} \mathrm{Zu}$ den amerikanischen Richtlinien von Information Control hinsichtlich deutscher Filme vgl. Bericht 40, Anm. 317, und Peter Pleyer, Deutscher Nachkriegsfilm 1946-1948, Münster 1965, S. 24f. Zur weiteren Entwicklung des Verhältnisses zwischen Sojusintorg und amerikanischer Militärregierung vgl. Bericht 10.

${ }^{66}$ Handschriftliche Bemerkung von Colonel William S. Paley auf dem zu diesem Bericht gehörigen Laufzettel: „Reference $8 \mathrm{~b}$. Russian officers are first to make examination and then make formal request for the loan of material needed by them". Wie die technischen Schwierigkeiten des Transports bewältigt wurden, konnte nicht ermittelt werden; die Kostüme kamen jedenfalls in Berlin an. Paley gehörte als Stellvertreter McClures der PWD an und war kurze Zeit nach der Auflösung von SHAEF stellvertretender Direktor von ICD USFET. Vgl. seine Memoiren: As It Happened, Garden City, New York 1979, S. 154171. 
sollten, Möglichkeiten für Aufführungen zu finden. Der Dirigent des Orchesters, Leo Borchard, scheint politisch einen einwandfreien Ruf zu haben. Er hat bisher elf Konzerte gegeben. Maßnahme: ein geeigneter Manager für das Orchester sollte zum frühest möglichen Zeitpunkt lizenziert werden, und dem Orchester sollte geholfen werden, damit es seine Konzerte fortsetzen kann. Leutnant Alter hat bereits mit den Special Services vereinbart, daß das Orchester im Titania-Palast üben und dort seine Instrumente lagern kann.

9. Dr. Van Eerden und ich besichtigten das Haus des Rundfunks gemeinsam mit Leutnant Gerlt von der Rundfunk-Abteilung, der einen Passierschein hatte. Der russische Wachhabende Hauptmann mit Namen Rosanow erklärte äußerst charmant, daß er es sehr bedauere, uns den Zutritt verwehren zu müssen. Sämtliche alliierten Offiziere müßten jedoch zwischen 19 und 20 Uhr anrufen, damit sie dann vom diensthabenden russischen Offizier einen Passierschein erhalten könnten. Er meinte, es sei ihm klar, daß das Studiogebäude sich im britischen Sektor befände, er sei aber von den britischen Wachen nicht abgelöst worden und sei nun an seine Befehle gebunden.

10. Dr. Rudolf Goldschmidt wurde als künftiger Verleiher befragt. Er war vorher bei MGM angestellt und scheint über die Fähigkeiten zu verfügen, die für diese Tätigkeit erforderlich sind. Wenn er von Oberst Leonard bestätigt wird, wird er unser Verleiher.

11. General McClure besuchte um 17 Uhr in Begleitung von zehn Personen das „Kabarett der Komiker“. Dieses Ensemble wurde von der NAAFI aus ihrem ständigen Haus im britischen Sektor hinausgesetzt. In der Aufführung war auch der berühmte Schlager „Berlin kommt wieder“ zu hören - erstaunlich gut von Brigitte Mira $^{67}$ vorgetragen. Als wir das Foyer betraten, stießen wir auf einen Hauptmann der USGCC, der das Management davon unterrichtete, daß dies die letzte Aufführung in dem Theater gewesen sei, in dem das Kabarett gerade „Onkel Toms Hütte“ spielte. Die USGCC entfernt alle Deutschen aus dem Gebiet.

12. Das Konzert der Berliner Philharmoniker um $18 \mathrm{Uhr}$ fand an einem Ort statt, der nicht gerade vielversprechend aussah. Die gesamte Vorderfront des Hauses ist zerstört. Als wir um das Haus herumgingen, sahen wir, daß das Orchester auf einer weiten Terrasse Platz genommen hatte. Ungefähr 1000 Stühle standen auf dem Rasen. Es waren etwa sieben- bis achthundert Zuhörer anwesend. Borchard ist ein mittelmäßiger Dirigent, das Orchester selbst ist ausgezeichnet. Das Programm begann dieses Mal mit der unvermeidlichen „Hebriden-Ouvertüre“ von Mendelssohn, es folgten die „Moldau“ von Smetana und Beethovens sechste Symphonie.

13. Am Vormittag des 19. Juli besichtigte ich eine Ausstellung zeitgenössischer deutscher Künstler, die den Nazis nicht genehm waren. Die Ausstellung wurde von der Kammer der Kunstschaffenden arrangiert und dort auch gezeigt. Ein Raum war

${ }^{67}$ Brigitte Mira, deutsche Sängerin und Schauspielerin. 
voll von Arbeiten von Carl Hofer. ${ }^{68}$ Er ist jetzt 70 Jahre alt, und sein gesamtes Werk ist zweimal dem Feuer zum Opfer gefallen. Die Gestapo hatte ihm verboten zu malen. Es gelang ihm jedoch, im geheimen zwischen 150 und 200 Bilder nachzumalen. Den 18 Bildern, die in der Ausstellung gezeigt wurden, sah man an, daß sie von einem Siebzigjährigen reproduziert worden sind. Der Bildhauer Paul Dierkes ${ }^{69}$ war durch einige hervorragende Skulpturen in Stein und in Holz vertreten. Eine Schnitzerei war aus einem tausendjährigen Balken gearbeitet; er stammte von der zerstörten Empore des Lübecker Doms. Die restlichen Arbeiten waren wenig originell und wenig eindrucksvoll. Die bemerkenswertesten Persönlichkeiten unter den Malern waren Ehmsen, ${ }^{70}$ der kürzlich in Rußland war, und Waldschmidt, ${ }^{71}$ ein ArbeiterMaler mit einer eher trockenen und formalen Art, der aber trotzdem eine starke Gestaltungskraft besitzt. Heinz Schwabe, ${ }^{72}$ der Vorsitzende der Abteilung für Bildende Kunst in der Kammer, entschuldigte sich für Ehmsen und Waldschmidt, indem er bemerkte, daß ihre persönliche Art vielleicht etwas massiv wirke.

14. Ich hatte eine Unterredung mit dem Vorsitzenden der Kammer, Paul Wegener, und erläuterte ihm die Konzeption, mit der wir die Kontrolle über Film, Theater und Musik auszuüben gedenken. Er fragte, ob die Konzessionen, die die Kammer erteile, ausreichen würden oder ob die amerikanische Armee zusätzlich zu den von der Kammer erteilten Konzessionen Einzelpersonen lizenzieren und registrieren würde. Ich sagte ihm, daß die Konzessionen allein nicht ausreichen würden. Er meinte auch, es sei ihm klar, daß Sonderregelungen für solche Personen getroffen werden müßten, die gezwungenermaßen der Nazi-Partei beigetreten seien; er führte als Beispiel seinen eigenen Sohn an, der, ohne der Partei beizutreten, nicht hätte Rechtsanwalt werden können, der aber, wie Wegener meinte, ,genau so denkt wie ich“. Ich sagte $i h m, d a ß$ wir in der Tat einen etwas strengeren Maßstab anlegten und daß Fälle von Personen, die gezwungenermaßen der Partei beigetreten sind, einzeln überprüft würden. Mein Eindruck von der Kammer ist, daß sie uns zwar nützen kann, daß man ihr aber nicht $\mathrm{zu}$ weitgehend vertrauen sollte. Auch scheint sie mir stärker daran interessiert, die Kunst-Szene von 1932 wiederzubeleben, als neue Dinge anzupakken. Wenn sie auf wirklich originelle Talente aufmerksam gemacht wird, kann sie eventuell hilfreich sein.

${ }^{68}$ Carl Hofer (1878-1955), Maler, 1920-1934 lehrte er an der Berliner Hochschule, deren Direktor er seit 1945 war.

${ }^{69}$ Paul Dierkes (geb. 1907), Bildhauer und Steinmetz, seit 1947 Lehrer an der Berliner Hochschule für bildende Künste.

${ }^{70}$ Heinrich Ehmsen (1886-1964), Maler, nach 1933 Mal- und Ausstellungsverbot, nach 1945 am Aufbau der Hochschule für bildende Künste in Berlin-Charlottenburg beteiligt, lebte nach 1950 in der DDR.

${ }^{71}$ Ludwig Waldschmidt (1886-1957), Maler und Graphiker.

72 Heinz Schwabe (geb. 1910), Graphiker, 1939-1945 Kriegsteilnehmer, Angehöriger der Widerstandsgruppe „Ernst“ in Berlin, 1946-1947 Lehrbeauftragter in Weißensee, lebt in München. 
15. Bei meinem letzten Besuch in Berlin war ich am Potsdamer Platz. Diesmal war ich zweimal am Potsdamer Platz und habe ihn beide Male erst nach einigen Sekunden wiedererkannt. Wenn Berlin wiederersteht, so wird das eine ganze Weile dauern.

$[\ldots]$

Report on Trip to Berlin, Davidson Taylor (Chief FTM ICD) to Intelligence Section ICD USFET, 20 July 1945; OMGUS 10/17-3/3.

\section{Henry C. Alter Halbwochenbericht, ${ }^{73}$ 21. Juli 1945}

\section{Film}

1. Verleih. In der Frage des Filmverleihs wurden beachtliche Fortschritte erzielt. Dr. Rudolf Goldschmidt wurde interviewt und probeweise als angestellter Verleiher für US-Filme im Berliner Distrikt bestätigt. Er wird noch überprüft und, falls vollkommen akzeptabel, bestätigt werden. Er wird sein Büro in der Nähe unseres Hauptquartiers einrichten und wird sofort in Verhandlungen mit Theaterbesitzern eintreten, um die ersten vier Theater zu bestimmen, in denen die Programme 1 und 2 des ursprünglichen PWD/SHAEF-Verleihplans um den 1. August herum gezeigt werden sollen. ${ }^{74}$ Es wird in Betracht gezogen, die vier größten zur Verfügung stehenden Theater in Betrieb zu nehmen; sie sind gleichmäßig über den US-Sektor verteilt, wodurch eine größtmögliche Verbreitung erzielt wird. Eine vorläufige Ubbereinkunft mit den Special Services stellt sicher, daß der Bedarf an Truppenkinos durch die Beschlagnahme von ungefähr acht Theatern gedeckt wird und daß nach dieser ersten Beschlagnahme der Verleih für das deutsche Publikum entsprechend geplant werden kann; für den Fall, daß weitere Beschlagnahmungen nötig werden, muß jedoch zunächst unsere Abteilung kontaktiert werden. Die Probleme, die im Zusammenhang mit den von Sojusintorgkino gemieteten Theatern aufgetaucht $\operatorname{sind}^{75}$, wurden in Besprechungen mit der russischen Kommandatura und dem Berliner Magistrat geregelt. Die Sojusintorg wird den russischen Sektor von Berlin weiterhin ebenso beliefern wie die gesamte russische Zone; sie wird sich aber aus den amerikanischen und britischen Sektoren von Berlin zurückziehen und auf ihren Anspruch auf die UfA und einige andere Theater in diesen Sektoren verzichten. Offenbar ist dieser Anspruch niemals wirklich ernsthaft erhoben worden, da er auf

${ }^{73}$ Handschriftliche Bemerkung - datiert vom 24. Juli 1945 - von Davidson Taylor auf dem zu diesem Bericht gehörigen Laufzettel: „Important, EXPEDITE“.

74 Die Programme bestanden aus zwei amerikanischen Dokumentarfilmen über Alltagsleben und Kultur in den USA und aus der neuesten Ausgabe der Wochenschau „Welt im Film“.

75 Vgl. Bericht 15, Anm. 102. 
der Annahme beruhte, daß diese Theater dem Berliner Magistrat gehörten; dem Magistrat hingegen war klar, daß ein solcher Schritt von den Besatzungsmächten hätte ratifiziert werden müssen und daß diese Ratifikation nicht erteilt worden wäre. Zur Zeit gibt es vier Typen von Theaterbesitz in Berlin: wirkliche UfA-Theater, erbaut, betrieben und noch im Besitz dieser Gesellschaft; sogenannte UfA-Theater, die von Juden oder anderen unerwünschten Besitzern requiriert worden waren; Theater, die vormals im Besitz bekannter Nazis waren und jetzt ebenfalls vom Magistrat übernommen worden sind; schließlich solche, die bis heute in der Hand des privaten Besitzers verblieben sind. Für unseren Verleihplan sind wir in einer Position, die es uns ermöglicht, aus allen vier Gruppen diejenigen Theater, die für unseren Zweck am geeignetsten erscheinen, auszuwählen. In den nächsten Tagen wird Dr. Goldschmidt überprüft, in sein Amt eingeführt und mit den nötigen Entscheidungsbefugnissen ausgestattet werden. Entscheidungen hinsichtlich der ersten vier Theater werden dann getroffen werden.

2. Produktion. Auch auf diesem Gebiet klärt sich die Lage etwas. Unsere Dienststelle hat Besprechungen mit den Vertretern der UfA Film $\mathrm{GmbH},{ }^{76}$ nämlich mit Herrn Feldes und Herrn Klatte, geführt. Diese Gesellschaft wurde 1942 als sogenannte „Dachgesellschaft" errichtet, die die gesamte Filmindustrie von der Herstellung des Rohfilmmaterials bis hin zur Kinoaufführung lenkte. Rechtlich kontrolliert diese Firma noch immer jedes einigermaßen bedeutsame Unternehmen in Deutschland, jedes Entwicklungslabor, sieben große Produktionsgesellschaften und zahlreiche Theater. Sie ist beim Berliner Gericht eingetragen, und der Finanzpräsident von Berlin ist ihr Vermögenstreuhänder. Die Gesellschaft hat eine Erklärung über ihren rechtlichen Status beigebracht; es sieht so aus, als sei sie, obwohl sie als das frühere Instrument der Nazi-Regierung vollkommen inakzeptabel ist, doch in der Lage, viele Fragen, die den gegenwärtigen Status der Filmindustrie betreffen, zu lösen. Für jede künftige Regelung müssen die Entscheidungen von Experten getroffen werden, trotzdem wird versuchsweise der folgende Vorschlag unterbreitet: Die Gesellschaft hat in Wirklichkeit drei Abteilungen: den künstlerischen und den Produktionszweig, bestehend aus den sieben Gesellschaften, die Filme produziert haben; den Bereich des Verleihs und der Theater und'schließlich den kommerziellen Apparat, der den gesamten technischen Bereich der Industrie kontrolliert: das Rohfilmmaterial, die Studios, Kameras, Entwicklungseinrichtungen usw. Offensichtlich müßten die beiden ersten Zweige von diesem letzteren abgelöst werden: Die Produktion wird von einem oder vielleicht zwei lizenzierten Produzenten übernommen, ebenso der Verleih von bestätigten und fest angestellten Verleihern, während die Kinos entweder in Privatbesitz bleiben oder als Staatseigentum konfisziert werden. Schließlich bleibt dann noch der Block von Gesellschaften und anderen Einrichtungen übrig, der möglicherweise die materielle Basis für die weitere Produktion in Deutschland bilden könnte. Was dann noch an technischer Ausrüstung vorhanden ist, könnte man dann zusammenkratzen, zusammenlegen und reparieren. Der Grundgedanke dabei

${ }^{76}$ Vgl. Bericht 1. 
ist, daß solche Anlagen dann an künftige Produzenten, die ausschließlich mit der künstlerischen Seite befaßt wären, vermietet und verkauft werden könnten. $\mathrm{Zu}$ diesem Zweck sollte der dritte Teil der gegenwärtigen und verflossenen UfA vielleicht erhalten werden, nachdem man vorher jedoch jede Verbindung sowohl mit der aktuellen Produktion als auch dem Verleih und dem Vorführbereich abgeschnitten hat.

Unsere Dienststelle wird ständig mit neuen Gesellschaften konfrontiert, die den Vorschlag machen, aktuelle Themen mit einem Minimum an technischer Ausrüstung produzieren zu wollen. Eine Gesellschaft, die sich selbst Kollektivfilm ${ }^{77}$ nennt, ist mit einer Bearbeitung fertig und besitzt einen Kontrakt mit der Tobis, einer der großen Tochtergesellschaften der UfA, in dem ihr Studio-Raum und Gerätschaften vermietet werden. Dieser Vertrag wurde jedoch mit dem Büro der Tobis geschlossen, während deren gegenwärtige Studio-Belegschaft mit Volldampf in den Johannisthal-Studios an russischen Synchronisationen arbeitet und die Abschlüsse des Hauptbüros völlig ignoriert. Eine andere Gesellschaft, die sich die „Deutsche Film Corporation" nennt, besteht aus früheren UfA-, Tobis- und Terra-Technikern, die ihre Talente und einige gerettete Gerätschaften zusammenwerfen wollen, um Filme zu produzieren und auch zu synchronisieren. Keine der beiden Gesellschaften kann als wirklich seriös angesehen werden, aber sie verweisen auf einen Trend, wonach Neugründungen wie Pilze aus der Erde schießen. Mit solchen Neugründungen werden wir es von jetzt an in zunehmendem Maße zu tun haben.

\section{Theater und Musik}

1. Theater. Es wurde entschieden, im amerikanischen Sektor mit der Lizenzierung von Theaterproduzenten zu beginnen. Um dieses Vorhaben zu erleichtern, muß für eine Zusammenarbeit der sechs Bezirks-Kulturämter gesorgt werden. Am Montag, dem 23. Juli, werden die Leiter dieser Ämter mit dem Kontroll-Offizier für Film, Theater und Musik zusammentreffen. Sie werden davon unterrichtet werden, daß vom kommenden Dienstag an alle Theatervorhaben, egal, ob es sich dabei um richtiges Theater oder um Kabarett handelt, und gleichgültig, ob sie bereits spielen oder erst proben, unserer Dienststelle gemeldet werden müssen. Es wird festgestellt, ob der Brief, der die Dienststellen der Militärregierung anweist, daß keine neuen Theaterprojekte gestartet werden dürfen, den deutschen Stellen bekannt ist. Diese Entscheidung wird ihnen gegebenenfalls noch einmal mitgeteilt. Dementsprechend werden Vorstellungen, die seit dem Erlaß dieser Entscheidung angelaufen sind, geschlossen, und die Produzenten werden angewiesen, unserem Büro Bericht zu erstatten.

77 Weder „,Kollektivfilm“ noch „Deutsche Film Corporation“ produzierte zwischen 1945 und 1948 Filme. Über die Produktionsfirmen, die zwischen 1945 und 1948 gegründet wurden, aber keine Filme fertiggestellt haben, konnten keine Angaben ermittelt werden. Eine Liste der deutschen Spielfilmproduktionsgesellschaften 1945-1948 bei Plever a. a. O., S. 459f. 
2. Musik. Dem Berliner Philharmonischen Orchester wird gemäß einer Vereinbarung mit dem Special Services-Offizier im Hauptquartier erlaubt im Titania-Palast zu proben sowie Instrumente zu lagern; die Philharmonie wird verwaltungsmäßig im amerikanischen Sektor von Berlin verbleiben. Ein Konzert des Orchesters für US-Truppen wurde von Special Services für Dienstag, den 24. Juli, arrangiert. Das Orchester wird ebenfalls im britischen Sektor vor britischen Truppen spielen und wird eventuell auch Gast-Konzerte in der Volksoper geben. Die Registrierung und Lizenzierung von musikalischen Aufführungen wird gleichfalls in der nächsten Woche beginnen.

Semi-Weekly Report No. 4, Henry C. Alter (FTM) to ISC Officer Berlin District, 21 July 1945; OMGUS 5/242-3/13.

\section{Henry C. Alter \\ Halbwochenbericht, 25. Juli 1945}

\section{Film}

1. Verleih. Die Úberprüfung von Dr. Rudolf Goldschmidt als Leiter des Filmverleihs im amerikanischen Sektor wurde heute abgeschlossen. Dr. Goldschmidt ist inzwischen Oberstleutnant d. L. Price ${ }^{78}$ vorgestellt worden, und es wurde gemeinsam beschlossen, das Vertriebsbüro im Gebäude der Kammer der Kunstschaffenden, Schlüterstraße 45, anzusiedeln. Die von den USA und Großbritannien geförderten Verleihfirmen werden je fünf Räume erhalten und zusätzlich können sie einen Projektionsraum und Schneideräume benutzen. In der Zwischenzeit wurden die Theater für die ersten Filmvorführungen in Berlin ausgesucht, es werden Vorbereitungen getroffen, um die Eigentümer bei der Militärregierung zu registrieren.

$[\ldots]$

Die Programmzusammenstellungen I und II des amerikanischen Verleihplans sind in Berlin angekommen, und die beiden Wochenschauen werden jeden Augenblick erwartet. Es könnte sich als notwendig erweisen, daß der Bürgermeister für das eine oder andere dieser Theater einen Leiter einsetzen muß, da die Eigentumsverhältnisse nicht mehr ganz klar sind. Ein Problem könnte die Stromversorgung werden, die zur Zeit oft bis spät in den Abend hinein unterbrochen ist. Verhandlungen mit dem Magistrat werden vielleicht notwendig, um eine einwandfreie Versorgung zu sichern.

Es kommen laufend Anfragen von Privatpersonen und Firmen, die im Filmverleihgeschäft mitarbeiten wollen. Ihnen wird mitgeteilt, daß es nur einen bevollmächtig-

${ }^{78}$ Wing Commander H. B. Price, Chief of Information Services Control for UK Sector Berlin. 
ten Verleiher geben wird und daß sie dessen Büro kontaktieren sollten bezüglich eventueller Beschäftigungsmöglichkeiten zu einem späteren Zeitpunkt, wenn sich der Verleih ausdehnt.

2. Produktion. Es muß unbedingt eine baldige Ưberprüfung der Akten der UfA Film $\mathrm{GmbH}$ vorgenommen werden, um den genauen Status dieser Firma und die Möglichkeiten zu ermitteln, wenigstens ein betriebsfähiges Spielfilmunternehmen zu retten. Die Kollektiv Film Co. ${ }^{79}$ hat in der Zwischenzeit Vorschläge für eine kleine unabhängige Produktion mit einfachsten Mitteln unterbreitet, für die sie das notwendige Kapital, die technische Ausrüstung und sogar ein Drehbuch zur Verfügung hat. Sie scheint ziemlich realistisch und findig zu sein und verdient möglicherweise einige Beachtung. Auf einer für heute einberufenen Konferenz mit Vertretern der UfA Tempelhof, ${ }^{80}$ einer Tochtergesellschaft des UfA-Konzerns, wird deren Status innerhalb der größeren Organisation ermittelt.

3. Filmvorführung. Nach den vorliegenden Plänen, die mit den Special Services und ISCS abgestimmt wurden, wird es sechs ständige Filmtheater für die amerikanischen Truppen und letztlich zwölf Filmtheater für die Zivilbevölkerung im amerikanischen Sektor geben. Damit ist die zukünftige Existenz von etwa zwanzig Theatern, die zur Zeit im amerikanischen Sektor spielbereit sind, in Frage gestellt, wobei die, die gerade hergerichtet werden, noch gar nicht berücksichtigt sind. Es wäre der Mühe wert, die Möglichkeit zu prüfen, ob einige dieser Filmtheater für echte Theaterzwecke und einige für die Filme verwendet werden können, die wir in Zukunft im Austausch von den britischen und russischen Verleihern erhalten.

\section{Theater}

Nach augenblicklich vorliegenden Informationen scheint es, daß es nur vier oder fünf Lizenzbewerber für die Aufführung von Schauspielen im amerikanischen Sektor geben wird. Die wichtigste Bewerbung, und in der Tat das einzig wirklich verfügbare Theater, wird das Hebbel-Theater ${ }^{81}$ in Kreuzberg sein. Der Leiter des Volksbildungsamtes ${ }^{82}$ dieses Bezirks, Herr Driesner, informierte unsere Dienststelle, daß nach dem jetzigen Spielplan die Aufführung der „Dreigroschenoper“ und von „Liliom" vorgesehen ist, zwei Stücke der leichteren Muse, die dem Geschmack des örtlichen Publikums entsprechen. Der Berliner Magistrat beabsichtigt jedoch für dieses Theater einen mehr klassischen Stil. Es ist erforderlich, daß ISCS bei der

${ }^{79}$ Zur Kollektiv Film Co. vgl. Bericht 10.

${ }^{80}$ Gemeint sind vermutlich die z. T. in Berlin-Tempelhof gelegenen technischen Einrichtungen des Ufi-Konzerns.

${ }^{81}$ Das Hebbel-Theater eröffnete am 15. August mit der Dreigroschenoper. Regie führte KarlHeinz Martin. Vgl. Bericht 20. Liliom von Franz Molnar wurde mit Hans Albers in der Hauptrolle erst am 25. April 1946 aufgeführt.

${ }^{82}$ Bei der Neubildung des Magistrats am 17. Mai 1945 wurde unter der Leitung von Stadtrat Otto Winzer (vgl. Bericht 1) eine Abteilung für Volksbildung eingerichtet, die in allen Berliner Bezirken Volksbildungsämter unterhielt. 
Lizenzvergabe für diese Theater seinen Wunsch äußert, welche der beiden vorgeschlagenen Stilrichtungen durchgeführt werden sollte. Ein weiteres großes Theaterprojekt besteht in den Bemühungen Jürgen Fehlings, ${ }^{83}$ der mit einer Truppe erstklassiger Schauspieler probt, allerdings zur Zeit noch ohne einen festen Theaterraum. Erkundigungen sind im Gange, und wenn das Projekt grundsätzlich akzeptiert werden kann, sollte Fehling in Betracht gezogen werden als der wahrscheinlich einzige in Berlin lebende Mann, der die Tradition Max Reinhardts ${ }^{84}$ fortsetzen könnte.

\section{Sitzung mit den Leitern von sechs Volksbildungsämtern}

1. Zweck. Eine Sitzung der Leiter der Volksbildungsämter aller Bezirke wurde von der Film-, Theater- und Musik-Unterabteilung für Montag, den 23. Juli, einberufen. Alle Leiter waren anwesend. Ziel dieser Zusammenkunft war es, den persönlichen Kontakt mit diesen wichtigen öffentlichen Vertretern herzustellen und ein System zu erarbeiten, nach dem sie ISCS in ihren jeweiligen Bezirken unterstützen können.

2. Allgemeiner Eindruck. Alle sechs zeigten großes Verständnis für unsere Absichten, sie zeigten auch große Eigeninitiative. Beispielsweise bei der Frage der Kabaretts erklärten zwei oder drei der Leiter, daß sie in einer so schweren Zeit wie der heutigen Kabaretts mißbilligten und daß sie selbst ihnen keine Genehmigung erteilen würden, zu spielen; nur einige wenige sehenswerte Kabaretts würden sie uns zur Genehmigung vorlegen. Diese sechs Herren scheinen ohne Ausnahme die richtigen Personen am richtigen Platz zu sein. Alle sind echte Antifaschisten, und sie würden Nazis oder solche, die zu erfolgreich unter dem Naziregime waren, nicht tolerieren. Einer von ihnen (Herr Stoll, Schöneberg) behauptet sogar, daß die Bevölkerung die „Favoriten“ der vergangenen zwölf Jahre nicht mehr sehen möchte und an völlig neue Gesichter und Ideen gewöhnt werden sollte, während andere wiederum eine etwas liberalere Haltung einnahmen und meinten, daß jedem, der in erster Linie Künstler, und zwar ein guter Künstler gewesen sei, erlaubt werden sollte, weiterzuarbeiten. Sie zeigten großes Verständnis für die amerikanischen Überprüfungsmaßnahmen und die Sammlung aller verfügbaren Informationen, deren Auswertung und Schlußfolgerung über Einzelpersonen.

3. Einstellung gegenüber der Kammer der Kunstschaffenden. Sie war weitgehend negativ. Sie glauben, daß, wenn diese Kammer überhaupt irgendeine Existenzberechtigung hat, sie für ganz Deutschland zuständig sein müßte. Zur Zeit wird die Kammer nur vom Berliner Magistrat unterstützt und ist viel zu stark aufgebauscht und ineffektiv, um ihre Existenz zu rechtfertigen. Es wurde darauf hingewiesen, daß die

\footnotetext{
${ }^{83}$ Jürgen Fehling (1885-1968), Regisseur, ab 1910 Neues Schauspielhaus Berlin, Märkisches Wandertheater, Volksbühne und Kammerspiele Wien, 1918-1922 Volksbühne Berlin, 1922-1945 Preußisches Staatstheater Berlin, 1945-1946 Leiter des Jürgen-Fehling-Theaters, 1949/50 arbeitete er in München, 1951 in Zürich, 1952 wieder in Berlin.

${ }^{84}$ Max Reinhardt (1873-1943), Theaterleiter, 1905-1920 und 1924-1933 Direktor des Deutschen Theaters in Berlin, 1938 Emigration in die USA.
} 
Hauptaufgabe der Kammer die Koordination der Interessen der drei Besatzungsmächte sein wird, und dies wurde eingesehen. Die gleichen Vorbehalte wurden hinsichtlich des Filmausschusses erhoben, die auch von unserer Dienststelle bereits angemeldet worden sind. Alles in allem scheint es, daß die Leiter der Volksbildungsämter glauben, mehr Verständnis für die örtlichen Probleme zu haben als die Kammer, und daß sie überzeugt sind, die kulturellen Aktivitäten selbst in die Hand nehmen zu können; aber sie akzeptieren die Kammer als zuständige Stelle für die Überwachungsmaßnahmen der Besatzungsmächte und deren Koordination.

4. Ergebnisse. Es wurde vereinbart, daß die Volksbildungsämter vollständige Listen aller kulturellen Programme und Aktivitäten vorlegen und alle Lizenzbewerber anweisen werden, sich in unserem Büro vorzustellen, wobei für jeden Bezirk ein spezieller Tag festgelegt wird. Es wurde außerdem vereinbart, daß die Leiter selbst vom ISCS eine Lizenz als Veranstalter solcher kleineren Veranstaltungen - Solistenkonzerte, Kammermusik, Dichterlesungen usw. - erhalten werden, wie sie ständig unter ihrer Schirmherrschaft stattfinden, mit der einzigen Auflage, daß solche Programme dem ISCS im voraus vorgelegt werden müssen. Abschließend wurde vereinbart, daß für ISCS bei allen Veranstaltungen und Programmen jeweils fünf reservierte Plätze vorgesehen werden.

Semi-Weekly Report No. 5, Henry C. Alter (FTM) to ISC Officer Berlin District, 25 July 1945; OMGUS 5/242-3/13.

\section{Henry C. Alter \\ Halbwochenbericht, 28. Juli 1945}

\section{Film}

1. Verleih. Die Eigentümer der vier Theater, die am Montag mit der Vorführung amerikanischer Filme beginnen, werden bei der Militärregierung registriert. Die Registrierung wird möglicherweise etwas verzögert werden, da Anfragen bei zwei Dienststellen der Militärregierung ergaben, daß sie mit den Registrierungsmodalitäten noch nicht vertraut sind. Es wird notwendig sein, die Antragsteller zu den Büros mitzunehmen und Registrierungen in jedem Bezirk zu veranlassen. Eine beträchtliche Werbekampagne hat eingesetzt, und Plakate, Zeitungs- und Rundfunkwerbung haben das Interesse an den Aufführungen vergrößert. Nach den allerletzten Nachrichten heißt es, daß Sojusintorgkino über die Eröffnung unserer Filmtheater sehr besorgt ist und daß sie ihre Verträge mit den einzelnen Theatern ernster nimmt als erwartet. Es bleibt abzuwarten, ob sie versuchen wird, sich in die Eröffnung der Theater am Montag einzumischen.

2. Produktion. Die UfA Film GmbH hat detaillierte Berichte und Aufstellungen vorgelegt, die so bald wie möglich von einem Experten geprüft werden sollten. Die 
Berliner Film-Arbeitsgemeinschaft (BFA) gehört zur jetzigen UfA-Organisation und gilt als Nachfolger der früheren UfA-Filmkunst, dem Unternehmen des großen UfA-Konzerns, das die Filme produzierte. Die UfA-Zentrale hat die BFA bevollmächtigt, alle technischen Einrichtungen zu sammeln und die Studios in Tempelhof betriebsbereit zu machen. Die Gründung der BFA geht angeblich auf das Mißtrauen der Russen gegenüber dem Namen UfA zurück. Die DFC oder „Deutsche Film Corporation “" ${ }^{85}$ eines der kleinen, wie Pilze aus dem Boden schießenden Unternehmen, behauptet, in der Lage zu sein, mit eigener Ausrüstung Filme fertigstellen und auch Filmaufnahmen ohne Ton machen zu können.

\section{Registrierung von Theater- und Musikveranstaltern}

1. Am 26. und 27. Juli schickten uns die Verwaltungsbezirke Zehlendorf und Steglitz die Lizenzbewerber für Theater- und andere Aufführungen. Zehlendorf schickte drei, Steglitz neun Personen, die Varietés, Kabaretts, Musicals usw. veranstalten wollen. Bis jetzt ist nicht einer dabei, bei dem sein jetziges Unternehmen eine Lizenz erfordert. Entweder sind die Bewerber nur Agenten, die bestimmte Varieté- oder Musical-Nummern übernehmen und damit Programme zusammenstellen, oder sie mieten einen Saal für diesen Zweck oder benutzen ihn mehr oder weniger ständig und ändern die Programme etwa monatlich. Gespräche mit den verschiedenen Leitern der Volksbildungsämter zeigen, daß sie selbst äußerst kritisch sind bei der Vergabe von Genehmigungen für diese Unternehmen und daß viele von ihnen ein sofortiges Aufführungsverbot zu erwarten haben. Aufgrund dieser Tatsache wird empfohlen, daß wir sorgfältig ein System ausarbeiten, nach dem jeder Verwaltungsbezirk ein wirklich anspruchsvolles Unterhaltungsetablissement unter der Schirmherrschaft und Mitwirkung des Volksbildungsamtes haben kann. Den Offizieren der Militärregierungscheint die Notwendigkeit dafür evident zu sein. OberstStahl(Steglitz) sagte sehr deutlich, daß er in seinem Bezirk ein Unterhaltungsprogramm für dringend notwendig hält, um wenigstens einige Leute von der Straße und von Dummheiten abzuhalten. In jedem Fall müssen wir warten, bis ein Überblick über alle sechs Bezirke vorliegt, und dann werden Maßnahmen ergriffen, um diese Unternehmen wirksam und zentral mit Hilfe der autorisierten Leiter der Verwaltungsämter zu kontrollieren. In der Zwischenzeit werden die Lizenzen für die Orchester vergeben, und dies sollte ohne große Verzögerung oder Schwierigkeiten geschehen.

Semi-Weekly Report No. 6, Henry C. Alter (FTM) to ISC Officer Berlin District, 28 July 1945; OMGUS 4/8-1/7.

85 Vgl. Anm. 77. 


\section{J. R. Foss ${ }^{86}$ \\ Reise nach Berlin, 31. Juli 1945}

1. Leutnant Alter konferiert und streitet mit den Briten über eine Art Oberste Kulturkammer, ohne die Russen zu berücksichtigen. Aufgrund früherer Erfahrungen glaube ich, daß es für uns eher nachteilig ist, dies zu tun, insbesondere wenn es um Verhandlungen mit den Russen geht. Ich habe festgestellt, daß die Russen bei einem von Briten und Amerikanern bereits gebilligten Vorschlag glauben, daß es zwei zu eins gegen sie steht und daß sie zu Anfang bei dem Problem nicht genügend berücksichtigt worden sind ${ }^{87}$ Ich glaube, daß umgehend jemand mit großem Einfühlungsvermögen nach Berlin gesandt werden sollte, um diese ersten Aktivitäten zu überwachen, damit wir nicht falsch beginnen oder in eine peinliche Lage geraten, weil wir eine Politik verfolgt haben, von der wir nur schwer wieder abgehen können.

2. Ich schlage vor, die gesamte Bremer Enklave dem Úberwachungsteam des Berliner Distrikts zu unterstellen, da ich nicht glaube, daß die zukünftigen Aktivitäten in Berlin das von uns vorgesehene Personal rechtfertigen würden. Wenn die Bremer Enklave aus dem Zuständigkeitsbereich des Frankfurter Teams herausgenommen wird, dann würde dieses von einem großen Problem und vielen Sorgen befreit. ${ }^{88}$

3. Ich schlage außerdem ein baldiges Zusammentreffen insbesondere mit der britischen und russischen Filmabteilung vor, da ich sehen kann, wo uns die Dinge entgleiten, wenn nicht umgehend etwas dagegen unternommen wird, und wo sich auch möglicherweise jemand auf die Zehen getreten fühlt, wenn wir die jetzige Situation aufrechterhalten, vor allem wenn russische Filme unter der ausschließlichen Kontrolle der Russen gezeigt werden.

4. Oberst Paley, Billy Wilder, ${ }^{89}$ Alter und ich sprachen über den Austausch von Filmen mit den Briten und Russen. Ich überredete Oberst Paley, zum gegenwärtigen

${ }^{86}$ Lt. Col. J. R. Foss war zu jener Zeit Deputy Chief von FTM ICD USFET.

${ }^{87}$ Später erging sogar eine Direktive des amerikanischen Kriegsministeriums, die sicherstellen sollte, daß gemeinsame amerikanisch-britische Aktivitäten nicht zu dem Schluß verleiten sollten, "that Germany has been divided into British-American and Russian sheres". NA RG 260 OMGUS 5/264 - 1/28: Message from Barrett, Washington, 11. 8. 1945. Die ursprünglichen amerikanischen Vorschläge für eine Viermächtekontrolle der deutschen information services ebenda, 5/264-1/9; vgl. auch 5/264-1/26.

88 Die Europäische Beratende Kommission hatte am 14. November 1944 die Bildung einer von amerikanischen Truppen zu besetzenden Enklave um Bremen innerhalb der geplanten britischen Zone beschlossen. Nach einer verwirrenden verwaltungstechnischen Praxis und verschiedenen Kompetenzstreitigkeiten wurde am 1. Januar 1947 das neue Land Bremen als viertes Land der amerikanischen Zone errichtet und OMGUS unterstellt. In Bremen wurde auch eine eigene ICD-Einheit stationiert. Im Originaldokument steht an dieser Stelle ein handschriftliches, vermutlich von Davidson Taylor stammendes „No“.

89 Billy Wilder (1906), amerikanischer Regisseur und Drehbuchautor österreichischer Herkunft, 1933 Emigration nach Paris, später Hollywood, drehte u. a. „Five Graves to Cairo“ (1943), „The Lost Weekend“ (1945), „A Foreign Affair“ (1948). „A Foreign Affair“ 
Zeitpunkt nichts in dieser Sache zu unternehmen, indem ich ihn überzeugte, daß wir nicht in der Lage sind, uns auf ein gemeinsames Programm festzulegen, bevor wir nicht mehr über die britischen und russischen Pläne wissen, die Leutnant Alter besorgen und für die morgen geplante Sitzung mit Major Coppock (Großbritannien) bereit haben wird.

5. Alle die bestehenden ,guten“ Theater liegen im britischen Sektor, und ich würde vorschlagen, daß wir versuchen, mit den Briten auszuhandeln, daß die ihnen von uns überlassenen Filme im Original in einem ganz bestimmten Theater gezeigt werden, das wir benennen. Ich bin ziemlich sicher, daß sie dem zustimmen würden, da sie sehr viele Theater in einer Straße haben.

6. Ich kann nicht eindringlich genug empfehlen, die Filmszene im Berliner Distrikt, insbesondere die Produktions- und Laboratoriumseinrichtungen und -möglichkeiten, unverzüglich und eingehend zu überprüfen, da ich glaube, daß viel gestohlen und geplündert wird. Außerdem glaube ich, daß sich die betroffenen Leute zusammengetan haben und alles tun werden, um - soweit sie können - uns Dinge wie Informationen und Materialien so lange vorzuenthalten, bis sie wissen, wie die Sache für sie ausgeht. Sie werden sehen, daß nach der Klärung der Lage die Leute in der Zone, die dem äußeren Anschein und ihrer Meinung nach die liberalste ist, plötzlich auftauchen werden.

7. Ich möchte nachdrücklich darauf hinweisen, daß das Berliner Überwachungsteam nur für das Berliner Gebiet Absprachen mit den Alliierten treffen kann. Während meiner Unterredungen mit unseren Leuten dort hatte ich den Eindruck, daß sie auf nationaler anstatt auf lokaler Ebene denken.

8. Wir müssen uns außerdem auch schnell entscheiden, was unsere Politik bezüglich Kabaretts, Nachtklubs etc. sein wird, wobei wir berücksichtigen müssen, daß diese Aktivitäten in den anderen beiden Sektoren erlaubt sind.

9. Ich schlage vor, den Deutschen in Berlin, die unserer Kontrolle unterliegen, ein vernünftiges und positives Programm zu präsentieren. Ich schlage vor, daß wir uns intensiv Gedanken machen über eine konkrete und konsequente Politik für Berlin auf lange Sicht. Die Leute, mit denen wir verhandeln müssen, sind sehr intelligent, und wenn wir ihnen diese Art von Programm unter Betonung einer klaren Politik bringen, bin ich sicher, daß wir mehr zum Entstehen des richtigen Verständnisses tun können, als alle diese offensichtlich falschen spontanen Aktionen erreichen, die unsere Verbündeten zur Zeit bieten und denen meiner Meinung nach jeder, der damit zu tun hat, sehr mißtrauisch gegenübersteht. ${ }^{90}$

spielte im Nachkriegs-Berlin und wurde dort zum größten Teil gedreht. Während der letzten Phase des Zweiten Weltkrieges produzierte er für das Office of War Information Propagandafilme. Anschließend arbeitete er kurze Zeit als Berater für Filmangelegenheiten bei ICD im besetzten Deutschland und drehte dabei den Film „,Die Todesmühlen“, einen Dokumentarfilm über Greueltaten in den NS-Vernichtungslagern. Überdies war er mit der Reorganisation der deutschen Filmwirtschaft befaßt.

90 Angespielt wird auf die „Information Control“--Politik der sowjetischen Militäradministration, die überwiegend aus ad-hoc-Aktionen bestand. 
10. Leutnant Alter hat ausgezeichnete Arbeit geleistet und tut es noch, aber er braucht unverzüglich noch einen gewichtigeren Mann an seiner Seite.

11. Ihr Telegramm wegen der Kostüme für die Berliner Oper ist angekommen. Um etwas Zeit zu gewinnen, habe ich Leutnant Alter angewiesen, sich von den Russen eine Aufstellung aller Berliner Kostüme geben zu lassen, die in dem besagten Bergwerk gelagert waren; ich informierte ihn, daß dies notwendig sei, da viele andere Opernhäuser ihre Kostüme ebenfalls in diesem Bergwerk lagerten. ${ }^{91}$ $[\ldots]^{92}$

Report: Recent Trip to Berlin and Munich, J. R. Foss (ICD USFET) to Davidson Taylor, 31 July 1945; OMGUS 10/17 - 3/3.

\section{Michael Josselson \\ Halbwochenbericht, 4. August 1945}

1. Besucherstatistik von vier Filmtheatern, in denen amerikanische Filme gezeigt werden:

\begin{tabular}{|c|c|c|c|c|c|}
\hline & & TIVOLI & PASSAGE & RIVOLI & COSIMA \\
\hline & Plätze: & 912 & 630 & 570 & 364 \\
\hline & $\begin{array}{l}\text { Bezirk: } \\
\text { Insgesamt }\end{array}$ & Tempelhof & Neukölln & Kreuzberg & Schöneberg \\
\hline 30. Juli & 10456 & 2477 & 3554 & 2949 & 1476 \\
\hline 31. Juli & 9609 & 2822 & 3274 & 2261 & 1252 \\
\hline 1. August & 9264 & 1892 & 3287 & 2246 & 1039 \\
\hline 2. August & 7148 & 1320 & 2763 & 1933 & 1132 \\
\hline 3. August & 6556 & 1002 & 2671 & 1624 & 1259 \\
\hline
\end{tabular}

An fünf Tagen insgesamt: 43033

Der Besucherrückgang von fast $40 \%$ innerhalb der kurzen Zeitspanne von nur fünf Tagen kann nur durch die allgemeine Aversion der Berliner Bevölkerung gegenüber dem ernsten Charakter der beiden gezeigten Programme erklärt werden. Ungeachtet der Tatsache, daß der Toscanini-Film weiterhin großen Anklang findet und daß sowohl die TVA und die Pipeline wie die „Fliegenden Festungen“ und die Brückenpanzer als Beweise für den unvermeidlichen Sieg der Alliierten bewundert werden, gibt es eine ständig wachsende Nachfrage nach amerikanischen Unterhaltungsfilmen. ${ }^{93}$

91 Das Telegramm konnte in den OMGUS-Akten nicht ermittelt werden. Es bezieht sich auf die in der Nähe von Eisenach gelagerten Kostüme. Vgl. Bericht 6.

${ }^{92}$ Der zweite Teil des Originaldokuments besteht aus dem Bericht über eine Reise nach München.

93 Gemeint sind Dokumentarfilme über den Dirigenten Arturo Toscanini, über die Tennessee Valley Authority (TVA), die in den 1930er Jahren ein System aus Wasserkraft und Sperren 
Die Zuschauer waren allgemein schockiert über die Tatsache, daß der ,große Maestro" "94 von den Faschisten verfolgt worden war. Große zynische Genugtuung wurde über das Schicksal der Japaner empfunden, das diese durch die Fliegenden Festungen erwartet. Die technologischen „Wunder“ der Amerikaner werden allgemein dem unbegrenzten Reichtum der Amerikaner zugeschrieben; das Sprengen von Brücken durch die Wehrmacht wird angesichts solcher Erfindungen wie der Brückenpanzer als ,idiotischer Wahnsinn“ hingestellt; die Filme vom Entlausungsverfahren, die allgemeine Heiterkeit hervorriefen, sind eingehend diskutiert worden, und zwar im Zusammenhang mit den in Berlin herrschenden unhygienischen $\mathrm{Zu}-$ ständen und der Notwendigkeit drastischer Maßnahmen gegen die krankheitserregende Fliegenplage und einer energischen Beseitigung der Leichen, die zu tausenden in den U-Bahn-Stationen liegen.

Die Vorführung der Programme erfolgte reibungslos, und es gab keine weiteren Berichte über Störversuche in den Projektionsräumen, wie sie den Vorführungen amerikanischer Filmprogramme unmittelbar vorausgegangen waren. Die US-Wachen, die am 28. Juli vor den vier Filmtheatern aufgestellt werden mußten, sind am 31. Juli zurückgezogen worden. ${ }^{95}$

Die Vertreter von Sojusintorgkino, Oberstleutnant Lipski und Oberstleutnant Fradkin, ${ }^{96}$ zeigten beim ersten Zusammentreffen mit den Vertretern unserer Abteilung großes ernsthaftes Interesse an einer Zusammenarbeit auf dem Filmvertriebssektor. Sie sind mit einer allseitig befriedigenden Lösung des Problems einverstanden, das durch die Verträge entstanden ist, die sie vor dem Einmarsch der amerikanischen und britischen Truppen in Berlin mit den Filmtheatern im gesamten Berliner Raum abgeschlossen haben, und auch durch ihren bestehenden Vertrag mit dem Berliner Magistrat über die Kinos der UfA-Kette. Eine Vier-Mächte-Besprechung ${ }^{97}$ über das Thema Filmvertrieb in Berlin wurde für die Woche nach dem 5. August in Aussicht genommen.

$[\ldots]$

zur Elektrizitätsversorgung errichtete, und über das Verlegen einer Pipeline im amerikanischen Westen. Die „Fliegenden Festungen“" war ein Dokumentarfilm über die Konstruktion von Langstreckenbombern und der Film über die Brückenpanzer zeigte Panzer beim Überqueren von Flüssen.

${ }^{94}$ Gemeint ist Arturo Toscanini (1867-1957), italienischer Dirigent, 1907-1921 an der Metropolitan Opera in New York, 1921-1931 an der Scala in Mailand, dirigierte bei den Bayreuther und Salzburger Festspielen, 1938 Emigration in die USA.

95 Bezieht sich auf Berichte über Sabotageakte an Projektionsausrüstungen in Filmtheatern des amerikanischen Sektors. In den OMGUS-Akten konnten die Berichte nicht ermittelt werden.

96 Oberstleutnant Fradkin war ein sowjetischer Vertreter beim Viermächte Information Control Committee.

97 Am 9. August beschloß die Alliierte Kommandatura auf ihrer 5. Sitzung, daß die Bühnenund Filmkontrollen in den einzelnen Sektoren Sache der zuständigen Kommandanten blieb. Alliierte Filme sollten zensurfrei sein und durch einen besonderen alliierten Filmverleih vermittelt werden. 


\section{Verschiedene allgemeine Informationen}

[...]

b. Thornton Wilders „Unsere kleine Stadt" 98 wurde nur kurz im Deutschen Theater gespielt. Nach zwei Aufführungen wurde es von den Russen abgesetzt. Oberstleutnant Sudakow schien sehr beunruhigt darüber, daß er das Stück ohne vorherige Zensur (?) zugelassen hatte und daß es sich nun als ungeeignet erwies. Obwohl die eigentlichen Gründe der Russen für diese Haltung bis jetzt nicht bekannt sind, ist das Stück, das zu den von den Amerikanern zugelassenen Stücken zählt, vielleicht deshalb unerwünscht, weil es einen gewissen Pessimismus verrät.

c. Ein russischer Film über Konzentrationslager (Majdanek und Auschwitz) soll in wenigen Tagen zur Aufführung in Berlin freigegeben werden. Von den Vertretern der Sojusintorgkino wurde der Vorschlag gemacht, ein gemeinsames Programm amerikanischer und russischer Filme über Konzentrationslager zu machen, um Wiederholungen zu vermeiden, die auf die Dauer dem Zweck der Vorführung solcher Filme entgegenwirken könnten. ${ }^{99}$

Semi-Weekly Reports No. 8B, Michael Josselson (ISCS Survey Sub-Section) to ISC Officer Berlin District, 4 August 1945; OMGUS 5/242 - 3/13.

\section{John Bitter, Henry C. Alter Wochenbericht, 4. August 1945}

\section{Film}

1. Umbesetzung innerhalb des Filmausschusses der Kammer der Kunstschaffenden. In einer der letzten Sitzungen gab die Kammer der Kunstschaffenden ein ermutigendes Zeichen dafür, daß sie in der Lage ist, ihre Probleme selbst zu lösen. Seit unserer ersten Untersuchung dort ist Paul Wegener darauf hingewiesen worden, daß wir von der Zusammensetzung des Filmausschusses nicht gerade begeistert sind. Man erklärte ihm, daß die Berufung bestimmter Personen ein temporärer Ausweg gewesen und teilweise unter Druck durch den Berliner Magistrat zugunsten gewisser Personen geschehen sei, die wir für kaum geeignet halten, zum gegenwärtigen Zeitpunkt solche Posten innezuhaben. Unsere Vorbehalte richteten sich hauptsächlich gegen zwei Herren: den Schauspieler Heinz Rühmann und den Produzenten Eberhard Klagemann, beide von der Terra Film. Es wurde ihm ferner erklärt, daß beide, obwohl wir keine schlüssigen Beweise gegen sie haben, zu sehr eine spezielle Firma

98 „Unsere Kleine Stadt“ hatte am 3. 8. 1945 in Max Reinhardts Deutschem Theater Premiere. Regie führte Bruno Hübner.

99 Der Vorschlag der Vertreter von Sojusintorgkino wurde nicht realisiert. Die amerikanische Militärregierung ließ einen eigenen Film - „Die Todesmühlen“ - produzieren. 
und außerdem den Filmboom repräsentieren, der unter Goebbels' Regiment in Deutschland herrschte. Die Kammer prüfte dann die ganze Angelegenheit und präsentierte uns eine vollständige Umbesetzung des Filmausschusses, bei der Rühmann, Klagemann, aber auch Erwin Schmidt, ehemals beim Deutschen Filmvertrieb beschäftigt, und Bruno Duday, ehemals bei der UfA, zum Ausscheiden gezwungen wurden. Alf Teichs und Dr. von Gordon bleiben für eine begrenzte Zeit. Unser Vorschlag, freigewordene Positionen neu zu besetzen, werde - so hieß es - befolgt werden. Es scheint, daß der Schauspieler Hans Söhnker, ${ }^{100}$ der zusammen mit Wegener als Nazigegner im Untergrund gewirkt hat und als solcher während der letzten Phase des Naziregimes von Gefängnis bedroht war, eine gute Wahl darstellen würde. Dies wird geprüft, und wenn Söhnker, der bisher wenig hervorgetreten ist, sich als geeignet erweist und sich dazu bereit erklärt, wird er sicherlich in den Filmausschuß berufen werden.

2. Produktionsunternehmen. Die Tobis, vertreten durch ihren Leiter, Herrn Reitzel, meldete sich im Laufe der vergangenen Woche und wird einen vollständigen Bericht $^{100}$ a über ihre derzeitige Organisation und ihr Vermögen vorlegen, einschließlich der von ihr gehaltenen Patente für das Fernsehen, einschließlich all ihrer wissenschaftlichen Forschung, und über ihre Studioanlagen in Johannisthal. Da letztere zur Zeit von den Russen für Synchronisationszwecke benutzt werden, kann die Tobis nicht vollständig darüber verfügen, aber sie hofft, daß diese Frage bis Ende der Woche geklärt werden kann. Ihr Vertrag mit der Kollektiv-Film wurde ebenfalls erwähnt, und Herr Reitzel schien optimistisch zu sein hinsichtlich eines Stoffes mit dem Titel „,Vor Tagesanbruch“, für den die Kollektiv-Film eine Produktionsgenehmigung erwerben möchte. Da festgestellt wurde, daß der Stoff einem Roman von Paul Ernst ${ }^{101}$ „entliehen“ ist, einem während des Naziregimes sehr beliebten Autor, wurde Reitzel darauf hingewiesen, daß dieses Projekt für uns nicht in Frage komme.

3. Vertrieb. In dieser Woche fanden Gespräche zwischen unserer Abteilung und Herrn John Levebre statt, der die Filmüberwachung von USFET und USGCC vertrat, ferner waren Major Frank Evans, Vertreter der Filmüberwachung bei der britischen Kontrollkommission, und Major Coppock, Vertreter der britischen ISCS in Berlin, anwesend. Herr Levebre besuchte die vier Filmtheater, die in Berlin eröffnet worden sind, und seine Vorschläge (die täglichen Vorstellungen auf vier zu reduzieren) wurden angenommen. Die Briten, so scheint es, werden ziemlich bald mit einem umfangreichen Programm beginnen und solange deutsche Filme vorfüh-

${ }^{100}$ Hans Söhnker (1903), dt. Schauspieler, spielte u. a. in den Filmen „Auf Wiedersehen, Franziska“ (1941, Regie: Helmut Käutner), „Große Freiheit Nr. 7“ (1944, Regie: Helmut Käutner). Söhnker scheint ein passiver Gegner des Nationalsozialismus gewesen zu sein, der ebensowenig wie Wegener im Untergrund tätig war. Vgl. Bericht 7.

${ }^{100 a}$ Der Bericht von der Tobis konnte in den OMGUS-Akten nicht ermittelt werden.

101 Das Projekt „Vor Tagesanbruch“ wurde nicht realisiert. Paul Ernst (1866-1933) schrieb Romane, Novellen und Dramen, u. a. Demetrios (1905), Brunhild (1909), Preußengeist (1915). 
ren, bis britische oder amerikanische Filme zur Verfügung gestellt werden können. Dies scheint auch für Berlin zu gelten, wo die Tobis, die ein Depot von ca. 70 Spielfilmen besitzt, vom britischen ISCS angewiesen wurde, diese für den britischen Filmvertrieb in Berlin bereitzuhalten, der entscheiden wird, welche der Filme dem deutschen Publikum gezeigt werden können. Es fand eine weitere Konferenz mit Oberstleutnant Lipsky, Oberstleutnant Fradkin von Sojusintorgkino und Herrn Billy Wilder statt. Es wurde vereinbart, daß der amerikanische Verleiher über den ISCS jedes beliebige Filmtheater im amerikanischen Sektor beanspruchen kann, selbst wenn dieses Kino nach dem gegenwärtig gültigen Vertrag mit dem Berliner Magistrat an Sojusintorgkino verpachtet ist. ${ }^{102}$ Die nächsten vier Filmtheater, die am 13. August eröffnet werden, wurden ihnen genannt, und eines davon steht auf ihrer Liste. Die endgültige Klärung wird erwartet. Gleichzeitig zieht die Sojusintorg die deutschen Filme aus ihren Filmtheatern in der amerikanischen Zone zurück. Dies wurde als Geste der Kooperation verstanden, da deutsche Filme eine größere Konkurrenz für die amerikanischen Filme bedeuten würden als russische. Es wurde außerdem vereinbart, daß die Frage der Eigentumsverhältnisse der UfA-Film-Theater und der Filmtheater ehemaliger Nazis auf höherer Ebene geklärt werden muß. Dabei wird gleichzeitig die Frage der Rechte der Sojusintorg in der amerikanischen Zone geregelt werden.

4. Filmvorführung. Die erste Besucherstatistik der vier Filmtheater, die zur Zeit amerikanische Filme spielen, zeigt, daß das Passage-Kino in Neukölln den meisten Zulauf hat und am ersten Tag 3554 Besucher zählte. Das Cosima-Filmtheater in Schöneberg weist folgenden ziemlich hohen Besucherrückgang nach dem ersten Tag auf:

$\begin{array}{ll}\text { 30. Juli } & 1476 \\ \text { 31. Juli } & 1252 \\ \text { 1. August } & 1039\end{array}$

Man muß am Montag die Programme der Filmtheater untereinander auswechseln, um einen etwa gleichen Besucherstand zu erhalten. Das Tongerät im Cosima-Filmtheater, das nicht gerade sehr gut war, wird überprüft und in einwandfreien Zustand gebracht.

Dr. Goldschmidt, der Verleiher, hat aus dem Zehlendorfer zivilen Fuhrpark einen Wagen zur Verfügung gestellt bekommen, und unsere Abteilung hat, ebenfalls in Zehlendorf, Büroräume für ihn gefunden.

\section{Theater, Kabarett und Varieté}

1. Registrierung des Theaterpersonals. Letzten Donnerstag wurde die Befragung und Registrierung für die sechs Verwaltungsbezirke abgeschlossen. Insgesamt wurden etwa 40 Personen befragt, Repräsentanten aller künstlerischen und semikünst-

102 Der Magistrat der Stadt Berlin hatte am 4. Juli 1945 die Berliner Lichtspieltheater an die sowjetische Filmverleihgesellschaft „Sojusintorgkino“ verpachtet. Vgl. Bericht 9. 
lerischen Unternehmungen, angefangen vom Hebbel-Theater und Berliner Kammerorchester bis hin zu kleinen Cafés und Einzelpersonen, die ein Violinkonzert finanziell unterstützen wollen. 21 Aufführungsräume wurden registriert, die größten mit 1500, die kleinsten mit 250 Plätzen. Mit Abstand das größte Kontingent stellen die Kabaretts dar, zu denen einige nichtständige Unternehmen zählen, sogenannte „Gastspieldirektionen“, deren Leiter Künstler oder Varieténummern engagieren und mit ihnen in verschiedenen Cafés, Sälen oder auch in solchen kleinen Theatern gastieren, die gerade frei sind. Die Gesamtzahl dieser Unternehmen beläuft sich auf 25. Bezogen auf die Verwaltungsbezirke ergibt sich folgendes Bild:

Zehlendorf: Gastspieldirektionen: 3

Erneuerungsantrag Vereinigung der deutschen Bühnenautoren und -verleger: 1

Steglitz: Gastspieldirektionen: 2

Kabarett: 1

Filmforschungsunternehmen (muß noch geprüft werden): 1

Schöneberg: Kabaretts: 9

Theater (Gastspiel-): 2

Beantragung einer Schauspielschule und eines Theaters: 1

Tempelhof: Kabarett: 1

Vorkehrungen durch die $\mathrm{VBK}^{102 a}$ für weitere Aktivitäten

Kreuzberg: Theater (Hebbel-Theater, Leiter: Karl Heinz Martin ${ }^{103}$ ): 1

Kulturtheater (Leiter: Cuno Fischer ${ }^{104}$ ): 1

Kabaretts: 4

Neukölln: Kabaretts: 6

Orchester sind in dieser Tabelle nicht berücksichtigt, sie werden getrennt unter Musik aufgefuihrt. Es ist offensichtlich, daß es im amerikanischen Sektor zu viele Kabaretts gibt. Die willkürliche Schließung einiger von ihnen würde jedoch nicht den Eindruck vermitteln, daß wir auch tatsächlich kontrollieren. Sie könnte nur als Diskriminierung durch uns angesehen werden. Es wird daher ein vorsichtiges Vorgehen ernpfohlen; man könnte zuerst einmal von den Kabaretts, die Tische anstelle von Sitzreihen haben, verlangen, keine gesprochenen Texte mehr zu bringen und sich auf Tanzmusik zu beschränken. In diesem Zusammenhang ist es interessant zu wissen, daß ein Tanzsaal, der einem der Varietétheater angegliedert ist und 700

\footnotetext{
102a Gemeint sind die Vereinigten Berliner Kunstbühnen.

${ }^{103}$ Karl Heinz Martin (1888-1948), vor 1914 Direktor des Komödienhauses in Frankfurt und Intendant des Frankfurter Schauspielhauses, nach dem Ende des Ersten Weltkrieges Mitbegründer der avandgardistischen Berliner „Tribüne“, arbeitete mit Max Reinhardt zusammen, 1927 Leiter der Berliner „Volksbühne“, 1932 Leiter des Deutschen Theaters und der Kammerspiele in Berlin, nach 1933 ging er nach Wien, nach 1945 inszenierte er zunächst am Berliner Renaissance-Theater, danach Intendant des Hebbel-Theaters.

${ }^{104}$ Cuno Fischer führte in der Premiere des Berliner Kulturkollektivs am 29. Juli 1945 mit $\mathrm{XYZ}$ von Klabund Regie.
} 
Personen fassen kann, jeden Tag nach zwei Stunden wegen Ưberfüllung zugeschlossen werden muß. Das dazugehörige Varieté hat 960 Plätze und wöchentlich durchschnittlich 5000 Besucher. Als nächster Schritt müssen sicherlich die Aufführungen besucht werden, um festzustellen, welche überhaupt von einem gewissen Wert sind. Dies sollte geschehen, bevor auch nur eines der Kabaretts, die Sitzreihen haben und keine Erfrischungen servieren, geschlossen wird.

Im Bereich des Theaters wird das Hebbel-Theater als das wichtigste Theater im amerikanischen Sektor untersucht, und Karl-Heinz Martin sind Antragsformulare für eine Lizenz zum Ausfüllen ausgehändigt worden, die sehr genau geprüft werden. $\mathrm{Er}$ ist vom Berliner Magistrat berufen worden, und wenn er unsere Überprüfungen nicht passieren sollte, wird es ratsam sein, die notwendigen Maßnahmen durch den Magistrat oder die Kammer der Kunstschaffenden vornehmen zu lassen. Rein künstlerisch ist Martin hoch qualifiziert, und seine Vorstellungen über den Spielplan, die er nach unseren Vorschlägen zu ergänzen bereit ist, sind vernünftig.

Ein weiterer Lizenzantrag wurde von der Scala eingereicht, die das NollendorfPalast-Theater gemietet hat und beabsichtigt, nach Abschluß der notwendigen Reparaturarbeiten dort zu spielen. Dies wird zur Zeit geprüft, und dem Bewerber wurde mitgeteilt, daß das Theater möglicherweise in zweifacher Hinsicht Verwendung finden muß, nämlich als Filmtheater und als Theater für die Scala. Die Scala war einst das größte Varieté- und Revue-Theater in Berlin.

\section{Musik}

1. Um zu einer Verständigung über das Programm der Berliner Philharmoniker zu kommen, wurde für den 31. Juli eine Sitzung in der Kammer der Kunstschaffenden einberufen. Offiziere der britischen und amerikanischen ISC, des Welfare und des Special Services sowie der Dirigent des Orchesters und dessen Geschäftsführer waren anwesend. Ein Acht-Wochen-Programm wurde verabschiedet, nach dem an jedem Wochenende zwei Konzerte abwechselnd einmal im Theater des Westens im britischen Sektor und einmal im Titania-Palast im amerikanischen Sektor stattfinden. Eines dieser beiden wöchentlichen Konzerte ist für die Truppen, das andere für die Zivilbevölkerung bestimmt. Das Orchester hatte bereits ernste Transportschwierigkeiten zu überwinden. Kesselpauken, Kontrabässe und ähnliche Instrumente mußten mit solch verschiedenen und primitiven Hilfsmitteln von Theater zu Theater transportiert werden wie Kinderwagen, Schubkarren, alte Maulesel und Rollschuhe. Das Konzert litt unter der physischen Erschöpfung der Musiker, so daß schließlich Vereinbarungen über die Beförderung der schwersten Instrumente auf Lastwagen getroffen wurden.

2. Die Überprüfung der Musiker, die von den Volksbildungsämtern zur Befragung geschickt wurden, wurde fortgesetzt. Die meisten guten Musiker kommen aus Schöneberg, wo die Stadtkapelle unter der Leitung von Dr. Coenen, das Blasorchester Schöneberg und das Schöneberger Kammerorchester voll organisiert und auf eini- 
germaßen anspruchsvolle Auftritte vorbereitet sind. Diese und kleinere Orchester spielen in Stadthallen, Kirchen und Schulen.

Zuversicht und Entschlossenheit sind kennzeichnend für die Einstellung der meisten Musiker, die befragt wurden. Einige der Musikgruppen konzertieren im Freien, nur um damit gleichzeitig spielen und üben zu können. Die meisten wurden ermutigt, diese Praktiken bis zu ihrer Lizenzierung beizubehalten. Hans von Benda, ${ }^{105}$ der Leiter des Berliner Kammerorchesters, darf bis zur Klärung der gegen ihn vorgebrachten politischen Anschuldigungen nicht dirigieren. Das Orchester wird am 11. August in Zehlendorf unter einem Gastdirigenten spielen. ${ }^{106}$

Die Lizenzgenehmigung für Leo Borchard, den Dirigenten der Berliner Philharmoniker, ist in Bearbeitung.

Weekly Report (In lieu of semi-weekly reports No. 7 and No. 8), John Bitter and Henry C. Alter (FTM) to ISC Officer Berlin District, 4 August 1945; OMGUS 5/242 - 3/13.

\section{John Bitter, Henry C. Alter, Edward Hogan Halbwochenbericht, 8. August 1945}

\section{Film}

\section{$[\ldots]$}

Ein Anteil von 50\% der Kasseneinnahmen für den Besitzer des Filmtheaters ist für Berlin sehr wenig, zumal Sojusintorgkino den Besitzern $60 \%$ gibt. Insbesondere wenn es nur eine begrenzte Anzahl von Vorführungen gibt ( 2 pro Tag, wie von ICD USFET gewünscht), ist dieser Prozentsatz für den Filmtheaterbesitzer ungünstig. ICD genehmigte versuchsweise mehr als zwei Vorführungen pro Tag, wenn es sich um kleine Filmtheater handelt und wenn kein Hauptfilm gezeigt wird. Für die vom Verleiher eingenommenen Gelder muß ein Sperrkonto bei der Militärregierung eröffnet werden, und ISCS wird das Recht haben, Geld abzuheben oder Abhebungen zu genehmigen. Sehr zum Ärger der Filmtheaterbesitzer liefert Sojusintorgkino weiterhin Filme in russischer Sprache in den amerikanischen Sektor. Für den britischen Sektor haben sie jedoch die Vorführung deutscher Filme nach einer Anfrage der Briten wieder zugelassen.

10.5 Hans von Benda (1888-1972), Generalmusikdirektor, 1935-1939 künstlerischer Leiter des Berliner Philharmonischen Orchesters, 1939-1945 Leiter des Berliner Kammerorchesters, 1948-1952 Leiter des Symphonieorchesters Valencia, Benda dirigierte am 13. Mai 1945 das erste öffentliche Konzert des Berliner Kammerorchesters im Schöneberger Rathaus.

${ }^{106}$ Das Berliner Kammerorchester spielte unter der Leitung von Sergiu Celibidache (1912). Celibidache, rumänischer Dirigent, studierte von 1939-1945 an der Berliner Musikhochschule, leitete von 1945-1950 die Berliner Philharmoniker. Er ist seit 1979 Generalmusikdirektor in München. 


\section{Filmarchive}

In einem - noch unbestätigten - Bericht eines Deutschen, der einige Monate lang bei unserem Intelligence Service gearbeitet hat, steht, daß die Russen etwa 5000 Kopien amerikanischer und britischer Unterhaltungsfilme, meist mit Untertiteln versehen oder synchronisiert, vom Babelsberger Lager des Reichsfilmarchivs ${ }^{107}$ weggebracht haben, außerdem noch deutsche Spielfilme jeden Alters, Filme über Konzentrationslager, die von den Nazis selbst gemacht worden sind, über Gaskammern, medizinische Experimente, ferner „Propaganda-Kompanie-Filme“ von Schlachten und neuen Waffen, deutsche, britische und russische Wochenschauen usw. Alle diese Filme sollen in die Geschäftsräume von Sojusintorgkino gebracht worden sein. Der Informant behauptet, daß alle zur Zeit von den Russen in Berlin gezeigten Filme, mit Ausnahme der allerneuesten, aus diesem Fonds stammen. Er berechnet den Wert der abtransportierten amerikanischen und britischen Filmkopien auf drei Millionen Dollar. Er behauptet, daß es an einer anderen Stelle (im russischen Sektor) noch ein weiteres, etwas kleineres Versteck gibt, das von den Russen bisher nicht entdeckt wurde. Er behauptet, daß er in einem Haus in Dahlem russische und deutsche Unterhaltungs- und Dokumentarfilme und einige wenige deutsche, französische und amerikanische Filme gefunden habe. Diese Lokalität soll von den amerikanischen Besatzungsbehörden zum Sperrgebiet erklärt worden sein.

\section{Theater}

1. „Unsere kleine Stadt“ von den Russen ,,verboten“. Am Samstag, dem 4. August, besuchte Herr Edward Hogan von unserer Dienststelle die zweite Aufführung des obengenannten Stückes im Deutschen Theater. Außer dem Beobachter saßen in der Mittelloge (ehemalige kaiserliche Loge) noch ein russischer Offizier mit einer Dolmetscherin. Der Genuß der Aufführung wurde dadurch beeinträchtigt, daß das Mädchen dem Russen das ganze Stück wörtlich übersetzte. Während der Pause waren der Offizier und das Mädchen verschwunden. Nach der Aufführung traf Herr Hogan mit dem Direktor Bruno Hübner ${ }^{108}$ und dem Intendanten Gustav von Wangenheim ${ }^{109}$ zusammen und erfuhr von ihnen, daß dies die letzte Vorstellung des Stückes gewesen war. Die russischen Besatzungsbehörden hatten - unter Hinweis auf eine Vereinbarung zwischen ihnen, den amerikanischen und den britischen Besatzungsbehörden - alle weiteren Aufführungen bis zur Klärung dieser dreifachen Zuständigkeiten verboten. Als offizielle Begründung wurde angegeben, daß die Aufführung völlig selbständig gewesen und weder gemeldet noch genehmigt worden sei. Das war die offiziell gegebene Version, aber Wangenheim deutete an, daß die

107 Das Reichsfilmarchiv wurde 1934 gegründet und ressortierte bei der Reichsfilmkammer.

108 Bruno Hübner (1899), dt. Schauspieler und Regisseur, 1934-1944 am Deutschen Theater, spielte u. a. in dem antijüdischen Film „Die Rothschilds“ (1940, Regie Erich Waschnek).

${ }^{109}$ Gustav von Wangenheim (1895-1975), Autor, Regisseur, Schauspieler, seit 1922 Mitglied der KPD, 1933-1945 Emigration in der UdSSR, Mitglied des Nationalkomitees Freies Deutschland, 1945-1946 Intendant des Deutschen Theaters. 
Ideologie des Stückes ein zusätzlicher Grund sein könnte. Der Inhalt ist zu passiv, zu poetisch und zu sehr „reine Kunst“, um den Russen, insbesondere zum jetzigen Zeitpunkt, zu gefallen.

Da das Stück, als Wangenheim die Leitung des Theaters übernahm, schon vorbereitet wurde, ließ er die Proben wahrscheinlich weiterlaufen, obwohl er bereits Schwierigkeiten auf sich zukommen sehen mußte. (Er ist Kommunist und hat die vergangenen 12 Jahre in Moskau gelebt.) Da das Stück nicht mehr gegeben wird, wurde das Problem der Tantiemen nicht weiter untersucht. Wangenheim hat eigene Vorstellungen bezüglich eines geeigneten Repertoires und ist gegen jede Art von reinem Kunsttheater oder reinem Unterhaltungstheater zum jetzigen Zeitpunkt. Er erwähnte speziell den „Mittsommernachtstraum“, dessen Aufführung er zum jetzigen Zeitpunkt nicht befürwortet. Seiner Meinung nach sollte jedes Theaterstück einen Anflug von Katharsis oder eines Schuldkomplexes enthalten. Er bat um amerikanische Schauspiele und erwähnte insbesondere „Die kleinen Füchse“, ${ }^{110}$ die er in Moskau gesehen hatte. Herr Hogan hatte sich persönlich dafür eingesetzt, dieses Stück in die vorläufig genehmigte Liste aufzunehmen, als diese in Washington und New York aufgestellt wurde, aber vergeblich, da es von Amerikanern des Jahres 1890 handelt, die nicht sehr nett sind. Es ist jedoch ein spezifisch antifaschistisches und außerdem sehr gutes Schauspiel. Herr Hogan hat sich für das Stuick eingesetzt, da er voraussah, daß es verlangt würde. Wangenheim gegenüber ist jedoch nichts von einer vorläufigen Liste freigegebener Stücke erwähnt worden. Das nächste Schauspiel am Deutschen Theater wird „Nathan der Weise“ sein, mit Paul Wegener als Nathan. ${ }^{111}$ In der ersten Septemberwoche wird Premiere sein. Das Theater selbst ist in sehr gutem Zustand, und sowohl der Geist wie die technischen Neuerungen seines großen Hausherrn früherer Jahre, Max Reinhardt, sind noch gegenwärtig. Demnächst wird es seinen Namen als „Max Reinhardts Deutsches Theater“ tragen.

2. Das Hebbel-Theater. Dieses Theater wird am Freitag mit der „Dreigroschenoper“ in der Bert Brecht/Kurt Weill-Fassung eröffnet. Die Lizenz für Karl Heinz Martin wird wahrscheinlich erteilt. ${ }^{112}$ Jedenfalls wird er eine Lizenz für diese erste Inszenierung erhalten.

3. Der Magistrat und die Kammer der Kunstschaffenden. Die Beziehungen zwischen diesen beiden Gremien haben sich in letzter Zeit verschlechtert. Herr Winzer vom Magistrat hat es für richtig gehalten, die Empfehlungen der Kammer zu ignorieren, und man weiß von ihm, daß er Ernennungen auf eigene Verantwortung vornimmt, ohne die Kammer auch nur zu konsultieren. Außerdem hat er keiner Besatzungsbehörde außer der russischen jemals irgendeine Frage zur Entscheidung vorgelegt, was Offiziere unserer Dienststelle veranlaßt hat, Vertreter der Kammer informell darauf

110 Das Theaterstück „Die kleinen Füchse“ stammt von der amerikanischen Autorin Lillian Hellman (1905). Das Stück wurde 1939 in New York uraufgeführt und 1941 unter der Regie von William Wyler verfilmt.

111 Das Schauspiel „Nathan der Weise“ hatte am 7. September 1945 Premiere.

112 Am 5. November 1945 erhielt Martin die endgültige Lizenz. 
hinzuweisen, daß es angebracht ist, uns jedenfalls immer dann zu konsultieren, wenn der amerikanische Sektor betroffen ist. Schließlich hat der Magistrat Paul Wegener, dem Präsidenten der Kammer, auch noch mitgeteilt, daß er mit der Wahl von Gericke, dem Geschäftsführer der Kammer, zum Mitglied des Präsidialrats der Kammer nicht einverstanden ist ${ }^{113}$ (Gericke wird von den amerikanischen und britischen Beobachtern als ausgezeichneter Mann für die Kammer mit gutem Urteilsvermögen eingeschätzt). Daraufhin bot Paul Wegener seinen Rücktritt an. Ein amerikanischer, ein britischer und ein russischer Kontroll-Offizier, die ohne Ausnahme in seinem Weggang einen großen Verlust für das kulturelle Leben Berlins sahen, mußten ihre ganze Überredungskunst aufbieten, um ihn dazu zu bewegen, seine Entscheidung neu zu überdenken und in die Kammer zurückzukehren, allerdings unter der einen Bedingung, daß er einen Assistenten bekommt, der ihm die Kleinarbeit abnimmt. Dieser Vorfall muß und wird als gemeinsame Aktion der drei Besatzungsmächte zur Unterstützung der Kammer gesehen werden.

\section{Musik}

1. In den musikalischen Kreisen Berlins gewinnt die Angelegenheit Hans von Benda, des Dirigenten des Berliner Kammerorchesters, immer mehr die Ausmaße einer "cause celebre“. Benda ist immer und überall anwesend, er hat ein gutes Mundwerk und kommt in dieser Stadt ohne Verkehrsmittel besser herum als die Leute der hier stationierten Panzerdivisionen. Er hat es irgendwie geschafft, einige Leute in wichtigen Positionen, einschließlich Mitglieder des Magistrats, zu überzeugen, daß er eine wichtige Stelle im Berliner Musikleben einnehmen sollte. Aus inzwischen erhaltenen Informationen geht hervor, daß seine musikalischen Darbietungen genauso unglücklich sind wie seine politischen, und daß der Stadt durch das „Spielverbot“, das ihn zur Zeit hindert aufzutreten, nicht viel verlorengeht. Dies gilt insbesondere, seit die beiden für den 8. und 11. August vorgesehenen Konzerte des Orchesters von einem talentierten und politisch unbescholtenen Rumänen namens Celibidache übernommen werden. ${ }^{114}$ Benda behauptet, Hitler habe ihn aus Bosheit zum Generalmusikdirektor ernannt.

2. Es ist über die Möglichkeit gesprochen worden, daß einige Dirigenten, die in den letzten Monaten nicht aktiv waren, ihre Arbeiten mit den Orchestern der Staatsoper und der Deutschen Oper wieder aufnehmen. Die beiden bekanntesten unter ihnen sind Robert Heger ${ }^{115}$ und Johannes Schüler. ${ }^{116}$ Beide sind fähig, versiert und erfahren. Beide waren anscheinend unpolitischen Geistes, obwohl sie, möglicherweise

$113 \mathrm{Vgl}$. Einleitung.

${ }_{114} \mathrm{Zu}$ Celibidache vgl. Anm. 106.

115 Robert Heger (1886), ab 1933 Staatskapellmeister an der Staatsoper Berlin, 1945-1950 Erster Kapellmeister an der Preußischen Oper Berlin-Charlottenburg.

116 Johannes Schüler (1894-1966), 1933-1936 Musikdirektor der Stadt Essen, 1936 1945 Kapellmeister an der Deutschen Staatsoper Berlin. 
gezwungenermaßen, Parteimitglied waren. Man hat ihnen nahegelegt, sich solange unauffällig zu verhalten, bis ihre Fälle überprüft sind.

3. Fünf Orchester- und sechs Solistenkonzerte sind für die Woche vom 5. bis 11. August vorgesehen, bis auf zwei alle im amerikanischen Sektor.

Semi-Weekly Report No. 9, John Bitter, Henry C. Alter, Edward Hogan (FTM) to ISC Officer Berlin District, 8 August 1945; OMGUS 5/242 - 3/13.

\section{John Bitter, Henry C. Alter, Edward Hogan Halbwochenbericht, 15. August 1945}

\section{Film}

1. Verleih. Die für den 13. August vorgesehene Eröffnung von vier Filmtheatern mußte um einige Tage verschoben werden, da die Filmkopien nicht rechtzeitig in Berlin eintrafen. Sie sollen nun bis Donnerstag eintreffen, und die neuen Filmtheater könnten eventuell am Freitag eröffnet werden. Die bereits laufenden Filmtheater spielen weiterhin Kurzfilme. Es wird immer schwieriger, einen Plan für die zukünftige Verteilung der Filme aufzustellen, da die für Berlin verfügbaren amerikanischen Filmkopien lediglich die Eröffnung von acht Kinos im amerikanischen Sektor zulassen. Das bedeutet, daß von insgesamt 45 Filmtheatern 8 amerikanische Filme zeigen werden, 6 Theater sind für den Special Services reserviert und 31 werden weiterhin mit Sojusintorgkino zusammenarbeiten, das ihnen russische und solche deutschen Filme liefert, die es für geeignet hält. Der amerikanische Verleih sollte endlich, um den amerikanischen Sektor kontrollieren zu können, in die Lage versetzt werden, alle Filmtheater im amerikanischen Sektor zu versorgen, selbst wenn freigegebene deutsche Filme gezeigt und deren Kopien aus der amerikanischen Besatzungszone beschafft werden müßten, in der davon große Mengen vorhanden sind. Von den in London freigegebenen Filmen ${ }^{117}$ und denjenigen Filmen, die von der Überprüfung eines Schauspielers abhängen, könnte eine gültige Liste aufgestellt werden - sofern bestimmte Schauspieler derzeit endgültig entlastet werden können -, wodurch die ärgsten Löcher in den amerikanischen Programmen gestopft werden könnten. Die Programme sollten immer durch eine amerikanische Wochenschau ergänzt werden,

${ }^{117}$ Das Office of War Information (OWI) und die Psychological Warfare Division (PWD) erstellten im Frühjahr 1945 eine Liste von Filmen, die sie für geeignet hielten, sie während der Besatzung den Deutschen zu zeigen. Viele der ausgewählten Filme waren während der NS-Zeit produziert worden. Ausgewählt wurden Filme, deren Inhalt und deren Darsteller politisch unbedenklich schienen. Vgl. auch Bericht 33. Bevor die amerikanische Militärregierung die Filme zur Benutzung freigab, waren diese noch von ICD USFET überprüft worden. Folgende Filme befanden sich auf der aus 27 Titeln bestehenden Liste: „Das Bad auf der Tenne“ (1943, Regie: Volker von Collande), „Komödianten“ (1941, Regie: Georg Wilhelm Pabst), „Meine Freundin Josephine“, „Das Mädchen von Fanö“ (Regie: 
von der ausreichend Kopien in Berlin gemacht werden könnten, wenn dies in München nicht bereits geschehen sein sollte.

2. Es ist von mehreren Fällen berichtet worden, wo Filmkopien - manchmal auch von amerikanischen Filmen - sich in Privatbesitz befinden. Dies wird überprüft, und die Filme werden beschlagnahmt und in die Schlüterstraße ${ }^{118}$ geschickt werden.

3. Die Deutsche Fox Filmgesellschaft hat mit unserer Dienststelle Kontakt aufgenommen bezüglich der Aufnahme des Verleihs ihrer eigenen Produktionen, von denen sie behauptet, daß sie einwandfrei seien. Sie wurde aufgefordert, Listen ihrer verfügbaren Negativfilme und Filmkopien vorzulegen.

4. Die UfA Film GmbH hat angefragt, ob es zur Zeit irgendwelche Pläne für eine deutsche Filmproduktion gibt. Wenn nicht, so sind sie gezwungen, das Unternehmen aufzulösen, da sie die Unkosten nicht länger finanzieren können. Es wird nochmals vorgeschlagen, daß ein Experte für Filmproduktionen ${ }^{119}$ zur Verfügung gestellt wird, der die von den verschiedenen Gesellschaften vorgelegten Berichte prüfen kann. Andernfalls sind wir verantwortlich für den Verlust von Werten, die wir zu unserem Vorteil hätten nutzen können.

5. Nach Aussagen der Filmtheaterbesitzer, die bereits die amerikanischen Kurzfilme zeigen, ist „Der Jeep“ der beliebteste Kurzfilm.

6. Es wurde zwischen der 82. Airborne-Division und unserer Dienststelle eine Vereinbarung über die Verwendung des UfA-Studios in Tempelhof getroffen, die folgendes beinhaltet:

Die 82. [Airborne-Division] verwendet ein Tonstudio und ein Bürogebäude für eine Schule.

Die UfA wird ein anderes Tonstudio und die angrenzenden Bürogebäude für ihre laufende Synchronisation russischer Filme benützen können.

Alle in den Studios beschäftigten Deutschen erhalten Ausweise, und anderen Deutschen ist der Zutritt verboten.

Die 82. [Airborne-Division] gestattet der UfA, alle Geräte und Ausrüstungen, die sie vielleicht auftreibt und irgendwo wiederfindet, in das Studio zu bringen. Wenn Ausrüstungsgegenstände weggebracht werden sollen, muß dies von unserer Abteilung genehmigt werden.

Die 82. [Airborne-Division] unterstützt die Arbeit der Zimmerleute und Metallarbeiter in den Werkstätten, sie wird dort selbst einen Teil ihrer eigenen Arbeiten erledigen und einige ihrer Leute zur „Ausbildung am Arbeitsplatz“ abstellen.

Hans Schweikart), „Operette“ und „Frauen sind keine Engel“ (Regie: Willi Forst). Vgl. OMGUS 10/11 - 1/19: Censoring German Films.

118 Die Büroräume der Kammer der Kunstschaffenden und des Amerikanischen Filmverleihs lagen ebenso in der Schlüterstraße wie die Lagerräume für die von FTM beschlagnahmten Filme.

119 Während des Winters 1945/46 wechselte die Besetzung dieser Position, deren Aufgabenbereich nicht klar umschrieben war, einige Male. U. a. waren Billy Wilder und Nicolas Nabokov angestellt. Erst nach der Berufung von Erich Pommer, früher Produzent der UfA, war die amerikanische Militärregierung in der Lage, den Wiederaufbau der deutschen Filmindustrie planmäßig zu forcieren. 
Die 82. [Airborne-Division] unterstützt außerdem die Reparaturarbeiten am

Tonstudio 3 und wird es, wenn es fertiggestelit ist, selbst verwenden. $[\ldots]$

\section{Theater}

1. Folgende Theater spielen zur Zeit im amerikanischen Sektor:

I. Das Hebbel-Theater, „Dreigroschenoper“, Premiere heute abend

II. Das Berliner Kulturkollektiv, zwei Einakter von Curt Goetz ${ }^{120}$

III. Das Freilichttheater, Stadtpark Schöneberg, „Der Widerspenstigen Zähmung" und „Im weißen Rössl“"

IV. Das Freilichttheater in der Argentinischen Allee, Zehlendorf, „Ein Mittsommernachtstraum" (eine Vorstellung)

V. Irgendwelche Aufführungen von Dramen sonntags in der Aula einer Schule; noch nicht überprüft

VI. Das Kleine Volkstheater, Kreuzberg

VII. Die Vereinigten Berliner Kleinkunstbühnen (die beiden zuletzt genannten Theater zeigen kleine Varieté-Nummern; bisher wurde noch keine Vorstellung besucht).

Außerdem gibt es noch 11 Kabaretts und Tingeltangel-Lokale mit Musik und Tanz, in denen offenbar außer den Bemühungen eines Conferenciers keine gesprochenen Darbietungen vorgesehen sind.

2. Herr Hogan hat die Militärgouverneure aller Bezirke im amerikanischen Sektor besucht, und alle haben folgendem Verfahren zugestimmt: Wir werden uns selbst nicht in die formelle Lizenzierung der verschiedenen Nachtlokale einmischen, aber wir werden sicherstellen, daß sie bei uns registriert sind, daß wir ihre Programme und die Art der Unterhaltung kennen, und daß sie mit der zuständigen Militärregierung die Eignung des Saales und andere öffentliche Sicherheitsvorschriften geklärt haben, für die die Militärregierung zuständig ist. Herr Hogan und Hauptmann Bitter werden in den nächsten Tagen die sechs Volksbildungsämter der verschiedenen Bezirke aufsuchen und ihnen erklären, daß sie unsere Lizenznehmer für die Überwachung, der zahlreichen erwähnten billigen Tingeltangel-Lokale sein werden.

3. An Davidson Taylor und Oberst Munsell ${ }^{121}$ ist unmißverständlich die Bitte herangetragen worden, für die Stücke „Thunder Rock“ von Robert Ardrey ${ }^{122}$ und „,On Borrowed Time" von Paul Osborn ${ }^{123}$ Aufführungsrechte zu erwerben. Man hofft. daß diese direkte Anfrage das New Yorker Büro der OWI veranlaßt, eine Art

${ }^{120}$ Das Berliner Kulturkollektiv spielte seit 12. August „Die Taube in der Hand“ und „Der Hund im Hirn“.

${ }^{121}$ Lt. Col. Warren P. Munsell war FTM officer bei ICD USFET.

122 Das Schauspiel ,Thunder Rock“ (Leuchtfeuer) von Robert Ardrey (1908) wurde erstmals 1939 in New York aufgeführt. Deutsche Erstaufführung war 1946 in Düsseldorf.

123 „On Borrowed Time“ (Galgenfrist) von Paul Osborne (1901) hatte 1938 in New York Weltpremiere. In Deutschland wurde das Stück 1946 in München zum ersten Mal gezeigt. 
Standard-Vereinbarung mit der Dramatist's Guild ${ }^{124}$ über die Rechte zur Aufführung dramatischer Stoffe durch Lizenznehmer in den besetzten Gebieten Deutschlands und Österreichs durchzusetzen. Karl-Heinz Martin vom Hebbel-Theater möchte die beiden genannten Stücke herausbringen, und zwar hat er ohne jeden Wink seitens unseres Büros darum ersucht. Das Stück von Ardrey steht auf der genehmigten Liste, das von Osborn wäre auch dabei, wenn man in New York nicht eine feste Liste von 25 Schauspielen verlangt hätte; durch diese Festlegung mußte man sich drastisch einschränken.

4. Es wird jetzt eine Vereinbarung getroffen, nach der das Theater am Nollendorfplatz (der ehemalige Mozartsaal) das von Amerikanern geleitete Erstaufführungskino mit einer Bühnenshow sein wird. Der zweite Teil des Programms wird - so hofft man - von einer Gruppe übernommen, die die alte Tradition der Scala für Berlin wieder aufleben lassen möchte. Die Scala war das berühmteste Varieté-Theater der Stadt, vielleicht sogar Europas.

\section{Musik}

1. Die folgenden Seiten enthalten eine Liste der Opernensembles, der Chöre und der wichtigsten Orchester im Berliner Raum. Die meisten Organisationen arbeiten unter extrem schwierigen Bedingungen, und es ist gut möglich, daß einige der schwächeren unter ihnen in absehbarer Zukunft aufgeben müssen.

2. Die Bedingungen für das Philharmonische Orchester bessern sich zunehmend. Nächste Woche wird der Vorstand in sein eigenes Büro in Zehlendorf ziehen. Dank der Zusammenarbeit mit Organisationen wie dem Berliner Zahnärzte-Orchester und anderen, inzwischen aufgelösten Orchestern wurden einige wertvolle Partituren unter Bergen von Trümmern und in verschiedenen Verstecken entdeckt. Dadurch ist die Orchesterbibliothek, die fast ganz verlorenging oder zerstört wurde, um einige wertvolle Partituren ergänzt worden. Einige Schlaginstrumente wurden auch noch gefunden.

3. Der Magistrat hat beschlossen, den Antrag des Steglitzer Städtischen Sinfonieorchesters auf finanzielle Unterstützung abzulehnen. Damit erhalten drei Orchester diese Unterstützung: das Staatsopernorchester, das Orchester des Deutschen Opernhauses und das Philharmonische Orchester.

\section{Opernensembles}

1. Die Staatsoper: Admiralspalast

Direktor: $\quad$ Ernst Legal ${ }^{125}$

Dirigenten: Karl Schmidt, Johannes Schüler

${ }^{124}$ Dramatist's Guild war gegründet worden, um die Urheberrechte der amerikanischen Bühnenautoren zu schützen.

125 Ernst Legal (1881), Intendant, 1933-1936 Schauspieler, Regisseur und Intendant am Theater in der Saarlandstraße Berlin, 1936-1945 Oberspielleiter am Schiller-Theater, Mai 
Erste Vorstellungen Anfang September, beginnend mit „Orpheus und Eurydike“ von Gluck. „Eugen Onegin“ von Tschaikowsky und „Rigoletto“ werden ins Repertoire aufgenommen. ${ }^{126}$

2. Das Deutsche Opernhaus: Theater des Westens

Direktor: Michael Bohnen ${ }^{127}$

Dirigenten:

Leopold Ludwig, ${ }^{128}$ Leo Spiess, Hajo Hinrichs, ${ }^{129}$ Robert Heger

Erste Vorstellung am 2. September mit „Fidelio“ von Beethoven. Eine geschlossene Vorstellung. Erste öffentliche Vorstellung am 4. September. ${ }^{130}$

\section{Die Kammeroper Bronsgeest: Städtische Bühne Friedenau Direktor: Cornelis Bronsgeest}

Ein kleines Ensemble von ungefähr 15 Sängern und 12 Instrumentalisten. Eröffnung am 16. August mit „Der Barbier von Sevilla“. In Vorbereitung: „Die lustigen Weiber von Windsor“" von Nicolai und „Der Wildschütz" von Lortzing.

Chöre

Mitglieder

früher heute

1. Kammerchor Waldo Favre

2. Chor der Berliner Philharmoniker

350

300

4. Chor der Berliner Singakademie

5. Ulmscher Dreifaltigkeitschor

6. Singgemeinschaft Lamy

7. Chor der Staatsoper

8. Chor des Deutschen Opernhauses
250

$150 \quad 70$

150 ca. 110

$120 \quad 80$

70 ca. 30

160

wird neu gebildet

$"$ " " " $"$

1945-Juni 1945 Intendant des Renaissance-Theaters, danach Leiter der ehemaligen PreuBischen Staatstheater, Intendant der Deutschen Staatsoper Berlin, 1949-1950 Mitglied der Provisorischen Volkskammer der DDR.

126 „Orpheus und Eurydike“ (Dirigent Karl Schmidt) hatte am 8. September, „Eugen Onegin“ (Dirigent Karl Schmidt) am 7. November und „Rigoletto“ (Dirigent Johannes Schïler) am 20. September Premiere.

127 Michael Bohnen (1887), Kammersänger, Mai 1945-Mai 1947 Intendant der Städtischen Oper Charlottenburg.

${ }^{128}$ Leopold Ludwig (1908), Generalmusikdirektor, 1936-1939 am Oldenburger Staatstheater, 1939-1944 I. Kapellmeister an der Staatsoper Wien und am Städtischen Opernhaus Berlin, nach Kriegsende Dirigent an beiden Berliner Opern und des Berliner Philharmonischen Orchesters.

${ }^{129}$ Hajo Hinrichs (1911), in den 60er und 70er Jahren Leiter der Opernklasse der Musikhochschule Hamburg.

${ }^{130}$ Die Vorstellung des „Fidelio“ vom 4. September leitete Robert Heger. 
Orchester

Anzahl der Musiker

früher heute Bemerkungen

1. Berliner Philharmoniker $120 \quad 100$ Dirigent: Leo Borchard. Die Konzerte sind ständig ausverkauft, ca. zwei Konzerte pro Woche.

2. Orchester der Staats- $140 \quad 100 \quad$ Gelegentliche Konzerte mit Gastdioper rigenten und den ständigen Operndirigenten.

$\begin{array}{llll}\text { 3. Orchester des } & 120 & 80 & \text { dito }\end{array}$

Deutschen Opernhauses

4. Orchester des $\quad 110 \quad 110$

Berliner

Rundfunks

5. Städtisches Sinfonieorchester Steglitz
Dirigent: Hermann Wunsch. Nur mäßiger Besuch bei den Konzerten. Ungewisse Zukunft.
6. Städtisches Sinfonieorchester Friedenau ca. $\quad 40$

7. Städtisches Orchester Schöneberg

Schöneberg

9. Berliner Kammerorchester
8. Blasorchester

Dirigent: Stallmann. Keine genaueren Angaben vorhanden. Zur Zeit keine Konzerte.

Dirigent: Dr. Coenen. Kleines Orchester. Konzerte 14tägig, manchmal zusammen mit dem Blasorchester Schöneberg. Siehe unten

Dirigent: Fritz Hubrig. Jeden Sonntag Freiluftkonzerte, manchmal auch zusammen mit dem Städtischen Orchester Schöneberg.

Dirigent: Hans von Benda. Gab ein Konzert mit mäßigem Erfolg unter der Leitung eines jungen Dirigenten namens CELIBIDACHE. Benda darf zur Zeit nicht dirigieren. 
Anzahl der Musiker

früher heute Bemerkungen

$\begin{array}{lll}\begin{array}{l}\text { 10. Kammerorchester } \\ \text { Friedrich Hain }\end{array} & 35 \quad \begin{array}{l}\text { Dirigent: Max Wenske. Gut, aber } \\ \text { gibt zur Zeit keine Konzerte. }\end{array}\end{array}$

11. Orchester Karl Kunze

60 Eine ad-hoc-Vereinigung. Keine genaueren Angaben vorhanden.

Semi-Weekly Report No. 11, John Bitter, Henry C. Alter, Edward Hogan (FTM) to ISC Officer Berlin District, 15 August 1945; OMGUS 5/242 - 3/13.

\section{Billy Wilder Propaganda durch Unterhaltung, 16. August 1945}

1. Zur Zeit eröffnen wir also nach und nach die Filmtheater in Deutschland wieder. Wir zeigen den Deutschen unsere Dokumentarfilme, einige Fakten, die sie kennenlernen und gut in Erinnerung behalten sollten. Wir zeigen ihnen Wochenschauen, die mit den Nachrichten gleichzeitig eine Lehre, eine Mahnung und eine Warnung verbinden. Zweifellos ist gute Arbeit geleistet worden. Die Deutschen sind im allgemeinen sehr empfänglich, und die allgemeine Reaktion ist positiv. Die Besucherzahlen schwanken zwischen ausverkauft und zufriedenstellend. Und doch wissen wir alle, daß es, wenn dieses Neue seinen Reiz verloren hat (in Berlin hat es seinen Reiz bereits verloren), immer schwieriger werden wird, ihnen so direkte Lektionen $\mathrm{zu}$ geben. Werden die Deutschen Woche für Woche ins Kino kommen, um den schuldbewußten Schüler zu spielen? Wir werden ihnen selbstverständlich unsere Spielfilme zeigen, die reine Unterhaltung neben den Dokumentarfilmen. Und sie werden natürlich kommen. Aber wir werden sie wahrscheinlich apathisch durch diese Dokumentarfilme und erzieherischen Wochenschauen dösen sehen - dann wach und bereit für Rita Hayworth in „Cover Girl“ ${ }^{131}$ „Cover Girl“ ist gewiß ein guter Film. Er hat eine Liebesgeschichte, er hat Musik und ist in Technicolor. Für unser Programm zur Umerziehung der deutschen Bevölkerung nützt er allerdings nicht viel. Wenn man nun aber einen Unterhaltungsfilm mit Rita Hayworth, Ingrid Bergman ${ }^{132}$ oder Gary Cooper ${ }^{133}$ machte, in Technicolor, wenn Sie wollen, und mit einer Liebesge-

131 Rita Hayworth (1918), amerikanische Filmschauspielerin, u. a. in „You'll Never Get Rich“ (1941), ,You Were Never Lovelier“ (1942) und - zusammen mit Gene Kelly „Cover Girl“ (1944) zu sehen.

132 Ingrid Bergman (1915), schwedische Schauspielerin, filmte u. a. in Deutschland und in den USA, spielte u. a. in „Wem die Stunde schlägt“ (1943) und „Das Haus der Lady Alquist" (1944).

133 Gary Cooper (1901-1961), amerikanischer Filmschauspieler, u. a. in „Wem die Stunde schlägt“ (1943) und „Mr. Deeds geht in die Stadt“ (1936). 
schichte - freilich mit einer ganz speziellen Liebesgeschichte, raffiniert gemacht, um ein bißchen Ideologie an den Mann bringen zu helfen - mit einem solchen Film hätten wir ein glänzendes Stück Propaganda in der Hand. Sie würden vor den Kassen Schlange stehen, und wenn sie ihn erst gesehen hätten, würde er haften bleiben. Leider gibt es diesen Film noch nicht. Er muß gemacht werden. Ich möchte ihn machen.

2. 1940 - Sie erinnern sich - während der schweren Luftangriffe auf London mußte den Menschen in den Vereinigten Staaten klargemacht werden, daß da ein richtiger Krieg im Gange war, daß England angeschlagen in den Seilen hing und dringend unserer Hilfe bedurfte. Die Wochenschauen leisteten ihren Beitrag, und wir waren von den englischen Dokumentarfilmen wie „Target for Tonight" ${ }^{\text {"134 }}$ ziemlich beeindruckt. Aber ich glaube, daß uns erst ein Hollywood-Film - ein „Unterhaltungsfilm" mit einer erfundenen Geschichte - wirklich gezeigt hat, was eigentlich los war. Und daß das, was der Familie Minniver in England geschah, auch leicht der Familie Jones in Iowa passieren konnte. Präsident Roosevelt hat denn auch, als er die erste Kopie von „Mrs. Minniver“ gesehen hatte, die Metro ${ }^{135}$ gedrängt, den Film so schnell wie möglich auf den Markt zu bringen. Und sie beeilten sich. Der Film hatte eine Wirkung, wie sie kein Dokumentarfilm und keine 50 Wochenschauen hätten haben können.

3. Filme wie „Mrs. Minniver“ oder der, an den ich denke, können nur von einer professionellen Filmgesellschaft gemacht werden. Sie müssen höchsten Ansprüchen genügen, was Technik, Drehbuch, Besetzung usw. betrifft. Ein solcher Film würde etwa $1 \frac{1}{2}$ Millionen Dollar kosten. Wir haben die Gruppe der Filmleute, die hier herumreist, ${ }^{136}$ während ihres Aufenthalts in Bad Homburg mit dieser Idee konfrontiert, und sie zeigten sich interessiert. Außerdem haben wir mit den HollywoodLeuten in Paris ${ }^{137}$ Gespräche über dieses Thema geführt. Die Herren Balaban und

${ }^{134}$ Der britische Durchhaltefilm „Target for Tonight“ (Ziel für heute Nacht) wurde 1940 von Harry Watts gedreht.

135 Die amerikanische Filmgesellschaft Metro-Goldwyn-Mayer Incorporated ging 1924 aus der Fusion der 1916 von Samuel Goldwyn gegründeten „Goldwyn Pictures C.“ mit den von Louis Burt Mayer gegründeten „Metro Pictures Cor.“ und „Louis B. Mayer Productions Inc.“ hervor. Die Metro produzierte 1942 mit „Mrs. Minniver“ - Regie William Wyler - einen der erfolgreichsten kommerziellen Propagandafilme.

136 Im Juli 1945 machte eine Gruppe von Repräsentanten der großen amerikanischen Filmgesellschaften eine Rundreise durch Europa und die amerikanische Zone im besetzten Deutschland - vorgeblich, um die amerikanische Militärregierung zu unterstützen, in Wirklichkeit aber, um nach dem amerikanischen Eigentum am europäischen Filmmarkt zu sehen und die Möglichkeiten für Hollywood auszuloten, den Verleih von amerikanischen Filmen in Deutschland zu monopolisieren. Während der Besatzungszeit 1945-1949 gelang dies in der Tat durch die Motion Picture Export Association (MPEA), die bis 1947 über die Militärregierung, ab 1947 in direkter Zusammenarbeit mit deutschen Lichtspieltheatern den Verleih amerikanischer Filme in Deutschland regelte. Vgl. auch OMGUS 10/ 11-1/27 und 28: Transfer of US Film Distribution to Industry (MPEA), Vol. I, II, ebenda, 10/17-3/5: Films: Technical Consulants. Vgl. Bericht 40.

137 In Paris saßen in dieser Zeit die Vertreter der großen Produktionsfirmen Hollywoods. 
Holman von Paramount ${ }^{138}$ meinten, daß Paramount mit diesem Film beauftragt werden sollte, da ich langfristig bei ihnen unter Vertrag und nur ,,vorübergehend an die amerikanische Regierung ausgeliehen bin“. Sie würden es als unfair ansehen, wenn ich ausscheiden und den Film mit einer anderen Gesellschaft drehen würde. Ich selber bin absolut dafür - ich habe neun Jahre lang nur mit Paramount gearbeitet, eine Reihe von Drehbüchern geschrieben und Regie geführt. Ich kenne den Stab und das Personal und ich glaube nicht, daß mir irgendeine andere HollywoodGesellschaft mehr Handlungsfreiheit geben könnte. Paramount ist bereit, mir TopStars, das beste Team und ein Budget von $1 \frac{1}{2}$ Millionen Dollar für den Film zur Verfügung zu stellen.

4. Wie Sie wissen, handelt es sich um die ganz einfache Geschichte eines GI, der hier bei den Besatzungstruppen stationiert ist, und eines deutschen Fräulein, oder, wie ich besser sagen sollte, einer deutschen Frau, da ihr Mann, ein Oberstleutnant bei der Luftwaffe, im Luftkampf über Tunesien gefallen ist. Ich habe eine solche Frau in Berlin getroffen - sie arbeitete in einer Kolonne, die den Schutt vom Kurfürstendamm wegräumte. Ich hatte eine Zigarette weggeworfen, und sie hatte den Stummel aufgehoben. Wir kamen ins Gespräch. Es verlief so: „Ich bin so froh, daß Ihr Amerikaner endlich gekommen seid, weil ...“ „Warum?“ „Weil Ihr uns helfen werdet, das Gas wieder in Ordnung zu bringen.“ „Das werden wir.“ „Das ist das einzige, worauf meine Mutter und ich warten ..." „Ich nehme an, es wird schön sein, wieder mal eine warme Mahlzeit zu bekommen.“ „Es ist nicht wegen des Kochens ..." Es entstand eine lange Pause. Ich konnte mir denken, was sie meinte, und hoffte, daß sie es nicht aussprechen würde. Aber sie tat es doch. „Wir würden es aufdrehen, aber nicht anzünden. Verstehen Sie? Wir wollen es nur einatmen, ganz tief.“ „Warum sagen Sie das?" „Warum? Weil wir Deutschen nichts mehr haben, wofür es sich lohnt zu leben.“ „Wenn Sie ein Leben für Hitler meinen, glaube ich, haben Sie recht." Ich hielt ihr eine ganz neue Lucky Strike hin. Sie nahm sie nicht. Sie nahm nur ihre Schaufel und ging zum Schutt zurück.

Gerade dieser kurze Dialog enthält das Thema des Films, und hier erkennt man das einfache Ziel, das ich erreichen möchte: wenn das Gas schließlich angedreht wird, zündet unsere deutsche Frau ein Streichholz an, um das Abendessen - nur ein paar kümmerliche Kartoffeln, zugegeben, - zu kochen, aber jetzt, nachdem ihr einige Dinge klar geworden sind, hat sie ,etwas Neues, wofür sie leben kann“. Das ist es, was der Film zeigen sollte (mit Eisenhowers Worten): „Daß wir nicht hier sind, um die Deutschen zu demütigen, sondern um zu verhindern, daß Krieg geführt wird“ und zum Schluß: „Gebt ihnen einen kleinen Hoffnungsschimmer, daß sie sich in den Augen der Welt rehabilitieren können."

Was den GI betrifft, so werde ich aus ihm keinen fahnenschwingenden Helden oder einen theoretisierenden Apostel der Demokratie machen. In Wirklichkeit möchte

${ }^{138}$ Die amerikanische Filmgesellschaft Paramount Pictures Corporation wurde 1914 gegründet. Später kam ein Wochenschauunternehmen, „Paramount News“, und ein Schallplattenunternehmen, ,Famous Music Cor.“, hinzu. Billy Wilder war von 1935-1954 bei Paramount unter Vertrag. 
ich, daß er zu Beginn des Films gar nicht so genau weiß, worum, zum Teufel, es in diesem Krieg überhaupt ging. Ich will die Fraternisierung, das Heimweh und den Schwarzmarkt mit hineinbringen. Außerdem soll der Mann (obwohl es eine Liebesgeschichte ist) das Mädchen nicht bekommen. Er geht mit seiner Division nach Hause zurück, während das Mädchen ,mit einem Hoffnungsschimmer“ zurückbleibt. All dies soll nicht pathetisch dargestellt werden. Lassen Sie mich einen anderen kurzen Dialog zitieren, den ich in Berlin hatte. Ich hatte einen deutschen Fahrer, und der Dialog zwischen uns spielte sich etwa folgendermaßen ab: „Diese Wahl in Großbritannien - jetzt wo dieser Attlee Churchill besiegt hat, was wird Churchill tun?“ „Ich nehme an, er bleibt in der Politik. Oder er schreibt ein Buch oder er malt.“ „Vielleicht wird er einen Putsch machen, nicht wahr?“ „Das glaube ich nicht.“ „Sie meinen, er wird nicht einmal Attlee erschießen wollen?" „Nein, das glaube ich nicht.“ „Sind Sie sicher?“ „Schauen Sie, Mann, Willkie ${ }^{139}$ unternahm keinen Putschversuch gegen Roosevelt, und Dewey ${ }^{140}$ hat Truman nicht erschossen.“ „Das ist komisch.“ „Es ist lustig, es ist demokratisch.“ Solche Sachen möchte ich in den Film hineinbringen, weil ich glaube, daß dies genau die richtige Art ist, Dinge zu sagen, ohne zu predigen.

5. Ich war zwei Wochen in Berlin, um einen Bericht über die zukünftige deutsche Filmproduktion zu schreiben. ${ }^{141}$ Ich sah eine verrückte, verkommene und hungernde Stadt, faszinierend als Hintergrund eines Films. Meine Notizbücher sind voll von brühwarmen Eindrücken. Ich habe jede Ecke fotografiert, die ich für die richtige Atmosphäre brauchen kann. Ich habe mit General Gavin, ${ }^{141 a}$ dem Kommandeur der 82. Airborne Division, jetzt die stärkste amerikanische Besatzungstruppe in Berlin, gesprochen: er hat mir jede Unterstützung zugesagt. Ich habe bei einigen seiner GIs gewohnt und ihren Jargon festgehalten. Ich habe mit russischen „Blitzmädchen" und britischen Militärpolizisten gesprochen. Ich habe mit Deutschen fraternisiert - vom ausgebombten Universitätsprofessor bis zu Drei-Zigaretten-Hürchen in der Femina-Bar. Ich habe beinahe meine Armbanduhr auf dem Schwarzmarkt unter dem Reichstag verkauft. Ich habe mir die Rechte an dem bekannten Lied „Berlin kommt wieder“ gesichert. ${ }^{142}$ Ich glaube, ich bin jetzt so weit, daß ich mich mit meinem Mitarbeiter zusammensetzen und das Drehbuch schreiben kann.

6. Der Film sollte nur in einer Fassung gedreht werden, in der die Amerikaner Englisch und gebrochen Deutsch sprechen, die Deutschen Deutsch und gebrochen Englisch, die Russen Russisch usw. Falls es nötig sein sollte, können gelegentlich

139 Wendell Lewis Willkie (1892-1944), amerikanischer Politiker (Republikaner), unterlag gegen F. D. Roosevelt in der Präsidentschaftswahl von 1940.

140 Thomas Edmund Dewey (1902), amerikanischer Politiker (Republikaner), 1943-1945 Gouverneur des Staates New York, verlor 1944 gegen Roosevelt und 1948 gegen Truman die Präsidentschaftswahl.

141 Vgl. Bericht 23.

141 a James M. Gavin (1907), war 1943 bei der Landung alliierter Truppen in Sizilien und 1944 bei der Landung in der Normandie beteiligt.

${ }^{142}$ Handschriftliche Randbemerkung von Paley: „Question whether this song should be used in the movie". 
Untertitel angebracht werden, aber ich will versuchen, alles so natürlich wie möglich zu halten. Wenn das Drehbuch fertig ist, soll es dem Kriegsministerium in Washington vorgelegt werden. Ich möchte außerdem der ICD eine Kopie zur Genehmigung zuschicken. Der Film soll in Hollywood gedreht werden, d. h. die Innenaufnahmen, die ungefähr $85 \%$ des Films ausmachen. Die Außenaufnahmen sollen in Berlin gedreht werden; sie werden ohne Ton gedreht, und es werden nur ein kleines Team von etwa 8 Leuten und die beiden Stars dort gebraucht werden. Ich schätze, daß wir etwa drei Wochen für diese Aufnahmen in Berlin bleiben müssen. Wenn die Armee den Transport in Europa nicht übernehmen kann, so könnte ich die Außenaufnahmen auch in Hollywood unter Verwendung von Rückprojektionen drehen.

7. Zusammen mit diesem Memorandum überreiche ich einen Bericht über die zukünftige deutsche Filmproduktion. Meiner Meinung nach ist die Produktion deutscher Filme in der nächsten Zeit nicht möglich. Es braucht einige Zeit, um die zukünftigen Produzenten zu überprüfen und die Schauspieler, Drehbuchautoren, Regisseure und Aufnahmeteams zusammenzubringen. Was die Ausrüstung betrifft, so ist das meiste gestohlen oder zerstört worden. Ich glaube, daß es in den nächsten acht bis zehn Monaten keine neuen deutschen Filme geben wird. ${ }^{143}$ Da wir nicht in Deutschland sind, um selbst Filme zu produzieren, sondern nur um die von Deutschen gedrehten Filme zu kontrollieren, habe ich in meinem Bericht vorgeschlagen, einen Mann zu finden, der in Berlin sitzen sollte und dessen Aufgabe, zusammen mit den Briten und Russen, darin besteht, alle vorgelegten Drehbücher zu lesen, ihre Linie zu prüfen und aufzupassen, daß keine faschistischen Ideen oder nationalsozialistische Propaganda in die Streifen kommt. Ich glaube nicht, daß mich die Abteilung für diese passive Aufgabe braucht. Was den Verleih anbelangt, so gibt es bei uns jetzt zwei äußerst kompetente Herren, Herrn Schwartz und Herrn Joseph, die solche Dinge sehr viel besser erledigen können als ich, der ich mich fast nie um etwas anderes gekümmert habe, als um das Machen von Filmen. Ehrlich gesagt: wenn ich noch länger in Bad Homburg bleiben sollte, hieße das, der Regierung Geld stehlen.

8. Wenn mir bald das Startzeichen für mein Filmprojekt gegeben würde, so könnte ich voraussichtlich Anfang nächsten Jahres den fertigen Film in General McClures Hände legen, um ihn der deutschen Bevölkerung zu zeigen. Ich bin eingebildet genug, zu behaupten, daß Sie in diesem „Unterhaltungsfilm“ die bisher beste Propaganda finden werden. ${ }^{144}$

143 Wilders Voraussage traf nicht ganz zu. „Die Mörder sind unter uns“ (Regie Wolfgang Staudte), der erste deutsche Nachkriegsfilm, wurde im Winter 1945/46 fertiggestellt und im Frühjahr 1946 uraufgeführt.

144 Wilder drehte in der Tat einen Film über Nachkriegs-Berlin mit dem Titel „A Foreign Affair“" (1948). Die Außenaufnahmen wurden z. T. an Originalschauplätzen gedreht. 
1. Herr Billy Wilder hat jetzt die Nachforschungen abgeschlossen, die notwendig waren, um mit dem Drehbuch und der Produktion des Films zu beginnen, den er in unserem Auftrag mit Paramount machen soll.

2. Es ist ein Memorandum vom 16. August 1945 mit dem Titel „Propaganda durch Unterhaltung" beigefügt, das mir Herr Wilder geschickt hat und in dem sein Vorhaben im einzelnen beschrieben ist.

3. Ich schlage vor:

a. Herr Wilder wird unverzüglich auf militärischen Befehl nach New York geschickt, um mit der Arbeit an diesem Film zu beginnen.

b. Er kann in New York zu OWI und privater Beschäftigung zurückkehren.

c. Unsere Abteilung bestätigt nach Erhalt und Genehmigung des Drehbuchs dem Kriegsministerium telegrafisch, daß diese Abteilung das Projekt unterstützt und daß sie die notwendigen Vorkehrungen zur Betreuung der 8 bis 12 Leute von Paramount, einschließlich Herrn Wilders, während der drei bis fünf Wochen übernimmt, die erforderlich sind, um die Außenaufnahmen in Deutschland zu drehen. ${ }^{146}$

18b. Stellungnahme Douglas Schneider für Oberst Paley (18. August 1945)

Ich stimme völlig mit Wilders Grundkonzept und mit Dave Taylors diesbezüglichen Empfehlungen überein.

Ich glaube, daß - vorausgesetzt General McClure stimmt den Empfehlungen zu einigermaßen schnelle Ergebnisse erreicht werden können.

Ich schlage vor, daß diese Unterlagen an General McClure zur weiteren Veranlassung geschickt werden.

P.S. Ich sehe einen Haken bei der Sache: Könnten wir eventuell Schwierigkeiten wegen Begünstigung der Paramount bekommen? Ich persönlich glaube es nicht, aber man sollte diesen Punkt im Auge behalten.

18c. Stellungnahme William S. Paley für General McClure (20. August 1945)

1. Ich schließe mich den Empfehlungen von Herrn Davidson Taylor an, die er im beiliegenden Memorandum gemacht hat. Vom Standpunkt unserer langfristigen Ziele aus gesehen, glaube ich, daß uns Herr Wilder äußerst wertvolle Dienste als

${ }^{145}$ Douglas Schneider arbeitete nach dem Zweiten Weltkrieg kurze Zeit in McClures Stab bei ICD.

146 Handschriftliche Randbemerkung von McClure: „The specific proposals for WD [War Department] help must be enumerated by Wilder and Paramount so we can fully advise WD Chief of Staff. M.“ 
Produzent des Films leisten wird, den er in seinem ebenfalls hier beigefügten Memorandum skizziert.

2. Herr Schneider wirft in seiner Stellungnahme die Frage auf, ob wir nicht vielleicht wegen Begünstigung der Paramount Company auf Kritik stoßen könnten. Es kann natürlich immer vorkommen, daß man für etwas kritisiert wird, was man getan hat, aber mir scheint, daß es in dieser Frage viele Präzedenzfälle gibt. Kommerzielle Filmgesellschaften haben sehr oft Kriegsfilme gedreht und dabei wertvolle Unterstützung von der Armee, der Marine und der Luftwaffe erhalten. ${ }^{147}$

Memo: Propaganda through Entertainment, Billy Wilder to Davidson Taylor (Chief FTM ICD USFET), 16 August 1945, recommendations D. Taylor 18 August 1945, William S. Paley 20 August 1945; OMGUS 10/16 - 2/15.

\section{Henry C. Alter \\ Halbwochenbericht (Film), 18. August 1945}

1. Es sind Gespräche mit Brigadegeneral R. A. McClure und Oberstleutnant F. N. Leonard bezüglich des Filmverleihs geführt worden; kurz zusammengefaßt hat sich folgendes ergeben:

a. Zur Zeit sind 52 Filmtheater im amerikanischen Sektor spielbereit, 48 sind tatsächlich in Betrieb, 8 davon zeigen amerikanische Filme, 6 stehen dem Special Service zur Verfügung. 34 Filmtheater werden demnach von Sojusintorgkino hauptsächlich mit russischen Filmen versorgt. Ungefähr $10 \%$ der russischen Filme sind Dokumentarfilme wie „Berlin“, „Wien“, „San Francisco“"148 usw., die auch von unserem Standpunkt aus den Deutschen gezeigt werden sollten. Der Rest sind russische Spielfilme, meist ohne Untertitel, und sie sind fast immer so schwach besucht, daß die Filmtheaterbesitzer nicht einmal ihre Unkosten decken können.

b. Dieser Zustand ist äußerst ungesund. Es wird daher die Erarbeitung von Spielplänen vorgeschlagen, die unserem Verleiher die Kontrolle über den gesamten amerikanischen Sektor und die Versorgung aller Filmtheater übertragen, auch wenn er sich die Filme von Sojusintorgkino besorgt, was er insbesondere bei Dokumentarfilmen und solch hervorragenden Filmen wie „Peter der Große“ usw. zweifellos tun wird. Nach diesem Spielplan würden die Filmtheater, die übrig bleiben, nachdem alle verfügbaren und geeigneten amerikanischen und russischen Filme eingesetzt worden sind, freigegebene deutsche Filme bekommen, und zwar in erster Linie die aus der genehmigten Liste des PWD Rear. ${ }^{149}$

${ }^{147}$ Handschriftliche Randbemerkung von McClure: „Col. Murphy - OK as amended“.

148 Gemeint sind sowjetische Dokumentarfilme über die Eroberung Wiens und Berlins durch die Rote Armee und über die Gründung der Vereinten Nationen in San Francisco.

${ }^{149}$ PWD Rear war bis zur Auflösung von Supreme Headquarters, Allied Expeditionary For- 
c. Dies ist insbesondere deshalb erforderlich, weil das britische ISCS 13 Filmtheater im britischen Sektor eröffnet hat, die alle deutsche Spielfilme zeigen. Ferner wird erwogen, daß wir vielleicht dieselben deutschen Spielfilme zeigen müssen, die im russischen und britischen Sektor freigegeben sind und gespielt werden, weil die Bevölkerung sonst Meinungsverschiedenheiten wittern und diese Filme dann erst recht in den entsprechenden Sektoren besuchen würde.

d. In diesem Zusammenhang ist es besonders wichtig, daß wir genügend Kopien der amerikanischen Wochenschauen für die Deutschen in Berlin direkt machen können, um damit alle Theater im amerikanischen Sektor zu versorgen. So wäre das Projekt erzieherisch sehr wertvoll, selbst wenn ein deutscher Hauptfilm gezeigt wird.

e. Die Frage der Kopien für diesen Plan ist noch nicht vollständig geklärt, aber es ist zu hoffen, daß für den Anfang genügend Kopien im Berliner Sektor bereitgestellt werden können, während wir dann für das weitere Programm vielleicht auf Kopien aus den Vorräten in der amerikanischen Besatzungszone zurückgreifen müssen. (Wächtersbach ${ }^{150}$ usw.)

f. Der Plan, den Nollendorf-Palast als amerikanisches Premierenkino mit einer guten Bühnenshow zu verwenden, ist ebenfalls diskutiert worden. Dieser von Feldwebel Winner von unserer Dienststelle vorgeschlagene Plan berücksichtigt die Tatsache, daß der Nollendorf-Palast zur Zeit von der „Neuen Scala“ wiederhergerichtet wird, einem Unternehmen, das aus dem ehemals größten Varieté-Theater Berlins hervorgegangen ist. Das Unternehmen hat beträchtliche Summen in den Wiederaufbau des Gebäudes investiert und einen Antrag auf Lizenzgenehmigung gestellt, um in diesem Haus das beste Varieté-Theater Berlins zu präsentieren. Der Antrag war einwandfrei, so daß man der „Neuen Scala“, wenn man bedenkt, wie viele Unternehmen gleicher Art, aber schlechterer Qualität existieren, die Genehmigung nicht gut verwehren konnte. Andererseits ist dieses Haus ein repräsentatives und großes Filmtheater, das an der Stelle liegt, wo der amerikanische Sektor am dichtesten an das Zentrum und auch an den britischen und russischen Sektor Berlins herankommt. Für unser Verleihprogramm liegt es daher äußerst günstig. Der „Winner-Plan“ berücksichtigt diese beiden Faktoren, und es ist nunmehr vorgesehen, den NollendorfPalast als Erstaufführungskino zu eröffnen, wo sich an eine erstklassige Bühnenshow ein besonders guter amerikanischer Film anschließt, der in Berlin anfangs nur dort gespielt wird. Ist er ungefähr einen Monat gelaufen, soll er abgesetzt und dann in den anderen Filmtheatern gebracht werden. Herr Robert Joseph ist mit diesem Plan einverstanden und hat den Film „Die gute Erde“ 151 als Eröffnungsfilm vorgeschlagen. Der Wert dieses Plans liegt vor allem darin, daß die amerikanischen Filme auf solche Weise von einem Publikum gesehen werden, das sie sonst nicht erreichen

ces (SHAEF) im Juli 1945 die Londoner Dienststelle der Prisoner of War Division (PWD).

${ }^{150}$ In Wächtersbach (Hessen) befand sich ein großes Filmlager der amerikanischen Militärregierung.

151 „Die gute Erde“ (The Good Earth) entstand 1937 unter der Regie von Sidney A. Franklin. 
würden, und zwar von all denjenigen, die in unmittelbarer Nähe des NollendorfPlatzes - gleich in welchem Sektor - wohnen, und von denen, die in erster Linie wegen der Bühnenshow kommen und vielleicht normalerweise niemals ein Filmtheater besuchen würden, in dem amerikanische Filme gezeigt werden. Mit den Scala-Leuten hat eine Besprechung stattgefunden; sie haben den Plan begeistert aufgenommen und werden uns in jeder Hinsicht unterstützen, wenn er genehmigt wird.

2. Am Freitag, dem 17. August, sind vier weitere Filmtheater im amerikanischen Sektor eröffnet worden und sie zeigen ein Programm von Kurzfilmen, während die vier Filmtheater, die am 30. Juli eröffnet haben, jetzt ihre ersten Spielfilme bekommen, und zwar „Pride and Prejudice“152، und „Der junge Tom Edison“. Diese Filme haben großes Interesse gefunden, und nach ersten Berichten waren alle Vorstellungen vollständig ausverkauft.

3. Ein Lager von Filmen aus dem ehemaligen Reichsfilmarchiv befand sich in einem Haus in der Hüningerstraße 52 in Dahlem. Der Verwalter dieses Hauses, ein Herr Griefe, steht zur Verfügung und könnte mit unserer Genehmigung das Material prüfen und dabei vielleicht einige Hinweise auf den Verbleib anderer Teile des Archivs finden.

$[\ldots]$

Semi-Weekly Report No. 12 (Film), Henry C. Alter (FTM) to ISC Officer Berlin District, 18 August 1945; OMGUS 5/242 - 3/13.

\section{Edward Hogan \\ Halbwochenbericht (Theater), 18. August 1945}

1. Am Mittwoch, dem 13. August, hatte am Hebbel-Theater vor ausverkauftem Haus die „Dreigroschenoper“ "Premiere, sie wird jetzt weitergespielt. Das Werk kommt einem in diesen Zeiten außerordentlich sarkastisch vor, aber es wurde gewählt, a) weil es ein beliebtes Stück und unter Hitler verboten war, und b) weil die Inszenierungsprobleme, jetzt wo das Material überall knapp ist, gering sind. Bei der Premiere gab es einen unerwünschten, aber nicht allzu schwerwiegenden Zwischenfall. Der zweite Akt schließt mit einem Song des Hauptdarstellers, den er vor dem Vorhang zu einem Chor hin singt, der ihm von der Galerie antwortet. Der Song hat einen Refrain, den man grob mit „Erst kommt das Fressen, dann die Moral“ übersetzen kann. Das wurde mit großem Beifall vom hungrigen Publikum aufgenommen. Der Unterzeichnete informierte den Direktor, Karl-Heinz Martin, daß wir den Refrain so abgefaßt haben möchten, daß er keine Demonstration auslöst. Bei der

152 „Pride and Prejudice“ (Stolz und Vorurteil) wurde 1940 gedreht. Die Hauptrolle spielte Laurence Olivier. 
dritten Vorstellung gestern abend (Samstag) schwächte der besagte Schauspieler den Refrain so ab, daß er vom Publikum kommentarlos aufgenommen wurde.

2. Weiteres zum Hebbel-Theater:

Am kommenden Mittwoch wird dort die Premiere von „Der Fröhliche Weinberg “153 von Carl Zuckmayer stattfinden. Ursprünglich war das Stück für das Babylon-Theater ${ }^{154}$ in der russischen Zone unter der Gesamtleitung von Martin vorgesehen. Wir vermuten, daß er das Stück in sein Haupthaus verlegt hat, weil die Amerikaner, seiner Ansicht nach, nicht so unangenehme Auflagen hauptsächlich bei der Úbersetzung machen wie die Russen. Die Russen verlangen immer eine russische Übersetzung all der Stücke, die in ihrer Zone aufgeführt werden sollen. Der Unterzeichnete wird - wenn möglich - die Generalprobe dieses Stückes besuchen.

3. Das Projekt Jürgen Fehlings (ein kleines Theater in unserem Sektor, das höchsten künstlerischen Maßstäben gerecht werden soll), wird sich leider beträchtlich verzögern, da unter den Mitgliedern des Ensembles die Ruhr ausgebrochen ist. Er plant als erste Aufführung den „Woyzeck“. ${ }^{155}$

4. Unser Plan, dem Scala-Unternehmen das Bühnenprogramm - die Hälfte des Gesamtprogramms - unseres Premierentheaters für amerikanische Filme (Nollendorf-Palast) zu überlassen, wird vorgelegt und wartet auf die offizielle Genehmigung (s. Bericht der Filmunterabteilung). Nachdem die Scala-Leute zuerst skeptisch waren, sind sie nun offensichtlich begeistert. Materielle Schwierigkeiten wegen der Reparaturen an diesem außerordentlich geeigneten Haus werden von der Militärregierung Kreuzberg behoben. Man ist der Ansicht, daß sich jeder Versuch lohnt, der dazu beiträgt, Besucher für die Filme anzulocken, die nach unserer Meinung von Deutschen gesehen werden sollten.

Semi-Weekly Report No. 12 (Theater), Edward Hogan (FTM) to ISC Officer Berlin District, 18 August 1945; OMGUS 5/242 - 3/13.

\section{John Bitter \\ Halbwochenbericht (Musik), 18. August 1945}

1. Am 16. August fand an der Städtischen Bühne Friedenau die erste Opernaufführung seit Kriegsende statt. Ein kleines Opernensemble, bekannt unter dem Namen „Kammeroper Bronsgeest“, brachte eine sympathische und redliche Aufführung der

${ }^{153}$ Regie führte Ernst Stahl-Nachbauer. Die Hauptrollen spielten Charlotte Radspieler und Franz Nicklisch.

154 Das Babylon-Theater am Schönhauser Tor spielte nur 1945/46. Am 1. Oktober hatte „Intimitäten“ von Noël Coward Premiere.

155 Das Jürgen-Fehling-Theater in Zehlendorf existierte nur in den Jahren 1945-1946. Es hatte am 27. August Gründungsfeier mit Szenen von Goethe, Shaw und Kleist. Büchners „Woyzeck“ gelangte nicht zur Aufführung. 
Oper „Der Barbier von Sevilla“ von Rossini. ${ }^{156}$ Was dem Ensemble und dem Orchester an Umfang und an Kraft fehlten, wurde durch Begeisterung und Mut kompensiert. Die Kostüme, das Bühnenbild und die Beleuchtung waren mehr als ausreichend. Das Haus war ausverkauft (625 Personen), das Publikum war restlos begeistert. Obwohl der Direktor, Cornelis Bronsgeest, während einer kurzen Zeitspanne Mitglied der Nazi-Partei war, haben sorgfältige Untersuchungen ergeben, daß er politisch einwandfrei ist.

2. Die Lizenzierungspraxis der Volksbildungsämter stößt auf einige Schwierigkeiten. Diese sollten innerhalb der nächsten Wochen beseitigt werden, aber aufgrund der stark beanspruchten Kommunikationssysteme scheint es ein langwieriger Prozeß zu werden. Dr. Heise und sein Assistent, Herr Balcke vom Schöneberger Volksbildungsamt, müssen genau beobachtet werden. Sie verkörpern eine Tendenz, in der mehr Wert auf Politik und Intrigen gelegt wird als auf die Förderung anständiger und lohnender Unternehmungen.

3. Auf der anderen Seite macht die Lizenzierung des Philharmonischen Orchesters gute Fortschritte. ${ }^{157}$ Alle Unterlagen sind inzwischen ausgefüllt und der IntelligenceAbteilung eingereicht worden.

4. Nach einem guten Start Anfang Juli ist die Beziehung zwischen dem TitaniaPalast und dem Philharmonischen Orchester auf gewisse Schwierigkeiten gestoßen. Alle Offiziere des Special Services, mit denen die Vereinbarungen getroffen worden waren, sind ersetzt worden. Einige Nachfolger haben die Existenz des Orchesters gefährdet, indem sie ihm nur Schwierigkeiten bereiteten. Es sind Maßnahmen getroffen worden, um dies zu korrigieren; aber wenn den Offizieren des Special Services, insbesondere den jüngeren, nicht klargemacht werden kann, daß ein so exzellentes Orchester wie die Philharmonie unbedingt erhalten werden muß, wird dieses kaum daran interessiert sein, in einem von Amerikanern kontrollierten Bezirk zu bleiben, sondern zu den Briten oder Russen überwechseln, wo sie mit offenen Armen aufgenommen werden.

Semi Weekly Report No. 12 (Music), John Bitter (FTM) to ISC Officer Berlin District, $18 \mathrm{Au}-$ gust 1945; OMGUS $5 / 242-3 / 13$.

156 Die Städtische Bühne Friedenau spielte nur 1945/46. Arthur Kusterer dirigierte „Der Barbier von Sevilla“. Cornelis Bronsgeest führte Regie.

157 Das erste Konzert der Berliner Philharmoniker hatte bereits am 26. Mai 1945 unter der Leitung von Leo Borchard im Titania-Palast stattgefunden. 


\section{Billy Wilder \\ Wiederaufnahme der deutschen Spielfilmproduktion, 21. August 1945}

1. Während meines Aufenthaltes in Berlin verschaffte ich mir einen Ưberblick über die Möglichkeit der Wiederaufnahme einer deutschen Spielfilmproduktion in Berlin. Im folgenden einige Fakten, die ich herausfand:

Die Studios in Berlin:

a. Das UfA-Studio Tempelhof, es heißt jetzt BFA (Berliner Filmatelier). Es befindet sich im amerikanischen Sektor. Die Bühnen sind in gutem Zustand. Es wurde leihweise vorübergehend der 82. Airborne Division für Laborarbeiten an Lehrfilmen und dgl. zur Verfügung gestellt.

b. Johannisthal (ehemals Tobis-Studios). Dieses Studio befindet sich im russischen Sektor. Die Bühnen 1, 3 und 5 sind benutzbar.

c. Die Althoff-Studios in Babelsberg im russischen Sektor. Die Bühnen 1 und 3 sind betriebsbereit. Die Bühne 2 wird repariert.

d. UfA-Studio Babelsberg. Es ist das größte Studio Europas. Da die Demarkationslinie zwischen dem russischen und dem amerikanischen Sektor mitten durch das Anwesen verläuft, ist die Sachlage etwas unklar. Dies ist alles, was ich in Erfahrung bringen konnte: Die Bühnen befinden sich alle im amerikanischen Sektor. (Es sind dies die großen Stummfilmbühnen, die vier Tonfilmbühnen und die beiden neuen Bühnen, die für Experimentierarbeiten gebaut worden waren, aber später für gewöhnliche Filme verwendet wurden.) Im russischen Sektor befinden sich die Werkstätten, die Holzvorräte, das Gebäude für Kulturfilme, die Filmlabors und die Stromversorgungsanlage. Die Herren, die diese Demarkationslinie festgelegt haben, würden sich sicherlich für eine neue Varieté-Nummer - das Zersägen eines lebenden Studios in zwei Hälften - eignen. Aber ich glaube, ich überdenke besser noch einmal das Wort „lebend“, denn das Herz und die Lungen sind herausgenommen worden. Die Russen haben die Stromversorgungsanlage und das Labor ausgeräumt und zusammen mit der gesamten übrigen Ausrüstung, die sie finden konnten, gen Osten geschickt. ${ }^{158}$

2. Es gibt sehr wenige Rohfilm-Vorräte in Berlin. ${ }^{159}$ Was es an Material gibt, ist von den Russen beschlagnahmt worden, sie behalten es nun für die Synchronisation ihrer Filme. Es gibt noch einige Stummfilmkameras aber fast keine Tongeräte. Die Gerüchte, daß noch enorme Mengen an Ausrüstung im amerikanischen Sektor vergraben seien, können nicht überprüft werden. Eine gute Planierraupe könnte die Antwort geben.

158 Zur Situation der Betriebsanlagen des Ufi-Konzerns in Berlin vgl. Spiker, a. a. O., S. 240.

159 Der Mangel an Rohfilm-Vorräten in der amerikanischen Zone und im amerikanischen Sektor von Berlin behinderte in den ersten Jahren der Besatzungszeit sowohl die deutsche als auch die amerikanische Filmproduktion. 
3. Ich hatte eine lange Unterredung mit Dr. Wolf von Gordon von der Kammer der Kunstschaffenden. Wie Sie wissen, arbeitet diese Kulturkammer für den Berliner Magistrat und wird von den Russen unterstützt. Sie ist eine Art Überwachungsinstitution für die Film-, Theater-, Musik- und Kunstszene in Berlin. Herr von Gordon ist der Leiter der Filmabteilung. Er hat folgendes gesagt: Die Kammer versucht, das Vertrauen aller Besatzungsmächte zu gewinnen und möchte für alle zukünftigen deutschen Produktionen verantwortlich sein, so daß diese in politischer und moralischer Hinsicht einwandfrei sind. Um jedoch eine solche Aufgabe durchführen zu können, muß man Vollmachten besitzen. Bisher hat nur der Magistrat der Kammer diese Vollmacht gegeben. Wenn zusätzlich alle Besatzungsmächte ihre Zustimmung geben würden, so könnten - nach Herrn von Gordons Meinung - enorme Fortschritte erzielt werden. Die Kammer würde die politische Zuverlässigkeit aller Personen der Filmbranche prüfen, ganz gleich ob sie Techniker oder Schauspieler, Regisseure oder Finanziers sind. Sie würde auch Filmprojekte prüfen, die dann natürlich noch einem Gremium der Alliierten vorgelegt würden, bevor sie zur Produktion freigegeben würden. Die Kammer ist sehr für private Produktionsgesellschaften. Herr von Gordon hält es nicht für notwendig, daß irgendeine der alten Gesellschaften wie die UfA, die Tobis, die Terra oder die Bavaria wieder auflebt. ${ }^{160}$ Er meint, in Wirklichkeit wäre es besser, sie in Vergessenheit geraten zu lassen, da sie zu eng mit der nationalsozialistischen Filmpolitik verbunden waren. Er ist gegen jede staatseigene und staatlich geprüfte Filmindustrie. Die Filmproduzenten und die Tendenzen der Filme müßten jedoch bei neugegründeten Gesellschaften auf ihre „politische Sauberkeit“ hin überprüft werden. Herr von Gordon meint außerdem, daß die Kammer irgendwann in Zukunft gerne den Vertrieb der Filme in Deutschland übernehmen würde. Die gesamte technische Ausrüstung für die Filmproduktion (mit Ausnahme der privaten Laboratorien) befindet sich jetzt in den Händen der Ufi ${ }^{161}$ der ehemaligen zentralen nationalsozialistischen Filmorganisation. Herr von Gordon findet das gut. Sie sollte zusammen mit all den Studios, Kameras, Apparaturen, Patenten, Lizenzen und dergleichen Staatseigentum bleiben. Die einzelnen privaten Gesellschaften müßten die Räume und die technische Ausrüstung von diesem staatseigenen Pool leihen. Diese Institution würde ohne Gewinn arbeiten. Sie könnte inflationistische Preise stoppen und für die Zuverlässigkeit oder die Produktion garantieren.

4. Ich hatte ein Gespräch mit zwei Mitgliedern der russischen Filmkommission: mit Oberstleutnant Fradkin und Oberstleutnant Lipski. Es wurde hauptsächlich über den Filmverleih in Berlin gesprochen, Leutnant Alter hat in seinen halbwöchentlichen Berichten darüber geschrieben. Als ich jedoch das Thema auf die Filmproduktion lenkte, merkte ich schnell, daß die Russen eine deutsche Filmproduktion für die Zukunft weder planen noch besonders daran interessiert sind. Die beiden Oberst-

160) Zur weiteren Entwicklung der ehemaligen Produktionsfirmen des Dritten Reiches vgl. Spiker, a. a. O., S. 240. Vgl. auch Der Spiegel vom 17.1.1951.

161 Vgl. Bericht 1. 
leutnants - Mitglieder von Sojusintorgkino, einer russischen Filmexportgesellschaft, die ein Monopol auf dem Berliner Filmmarkt hatte, bis wir und die Briten einmarschierten - versicherten mir, daß sie, obwohl sie Uniform mit Orden trugen, nur ein privates Unternehmen, das sich ausschließlich mit dem Verleih und der Vorführung von Filmen befaßt, vertreten. Alles, was sie zum Thema Filmproduktion zu sagen hatten, war, daß sie erwarteten, daß jeder Film, den die Deutschen drehen wollten, von allen Besatzungsmächten genehmigt werden müßte, unabhängig davon, ob der Film in unserem Sektor, in Berlin, im britisch besetzten Hamburg oder im russisch besetzten Breslau gedreht würde. ${ }^{162}$

5. Vorschlag:

Obwohl ich der Meinung bin, daß unser System der Überprüfung aller Filmleute, die wieder arbeiten möchten, und der Lizenzierung derjenigen, die politisch unbelastet sind, weiterverfolgt werden sollte, befürworte ich ein Viermächte-Filmgremium in Berlin, das nicht nur bereits vorhandene deutsche Filme - wie mit Fred Schwartz ${ }^{162 a}$ besprochen - zensiert, sondern auch Beurteilungen, Zustimmung oder Ablehnung von Filmprojekten - insbesondere zu Spielfilmen - abgibt. Spielfilme kosten sehr viel Geld, und wenn sie nicht in ganz Deutschland und Österreich gezeigt werden könnten, würde sich ihre Herstellung nicht lohnen. Kein Mensch kann es sich leisten, einen kostspieligen Film in München zu produzieren ohne sicher zu sein, daß er auch in Hamburg, Berlin oder Breslau gezeigt werden kann. Andererseits müßte ein im russischen Sektor gedrehter Film von uns genehmigt werden, bevor wir dessen Vorführung z. B. in Bayern zulassen würden. Ein derartiges Viermächte-Gremium, das reibungslos zusammenarbeitet, könnte für eine zukünftige deutsche Filmproduktion von größtem Nutzen sein. Ich schlage außerdem vor, daß unser DISCC-Team die Kulturkammer weiterhin überprüft. Ich habe gehört, daß sie die erste Garnitur der Mitglieder ,entlaust“ hat. Sie haben einen guten Eindruck auf mich gemacht, und ich sehe keinen Grund, warum wir die Kammer nicht für die Vorarbeiten bei Filmprojekten einsetzen sollten. Sie sollte dann dem Viermächte-Gremium die konkreten und von ihr bereits genehmigten Filmprojekte vorlegen. Was unseren Vertreter in diesem Viermächte-Filmgremium betrifft (das, wie ich annehme, auf der hohen Ebene der Group $\mathrm{CC}^{163}$ arbeiten würde), so schlage ich einen Mann vor, der nicht unbedingt Filmexperte sein muß. Ein politischer Mann mit gesundem Urteilsvermögen wäre genau der richtige Vertreter unserer Interessen.

\section{2a. Stellungnahme Davidson Taylors ${ }^{164}$ (28. August 1945)}

1. Ein Bericht von Billy Wilder an mich vom 21 . August 1945 mit dem Thema „Die Wiederaufnahme der deutschen Spielfilmproduktion“ ist beigefügt.

162 „Die Mörder sind unter uns“ - der erste deutsche Nachkriegsfilm - wurde vermutlich nur von der sowjetischen Militäradministration genehmigt.

162a Fred Schwartz war Chef der Film Distribution and Exhibition Branch von USFET ICD.

163 Gemeint ist die US Group Control Council (USGCC), Vorläufer von OMGUS.

${ }^{164}$ Handschriftliche Randbemerkung von Lt. Col. Adrian Murphy, Deputy Chief von ICD USFET: „FTM Section: A \& B approved for multipartite discussion. A. M.“ 
2. Er schlägt im Absatz 5 vor, ein aus allen vier Besatzungsmächten bestehendes Filmgremium in Berlin zu bilden, das sowohl deutsche als auch neutrale Filme prüft und Filmprojekte genehmigt.

Vorschlag: das betreffende Zensurgremium, das auf Viermächtebasis in Berlin eingerichtet werden soll, übernimmt auch die Verantwortung für die Weitergabe von Drehbüchern, die von lizenzierten Filmgesellschaften zur Verfilmung vorgeschlagen werden. ${ }^{165}$

3. Herr Wilder schlägt außerdem vor, daß die Kammer der Kunstschaffenden für die Vorarbeiten an den Filmprojekten eingesetzt werden sollte und dann dem Viermächte-Zensurgremium die Projekte vorlegt, die die Zustimmung der Kammer haben. Die einzige Gefahr, die ich darin sehe, ist die, daß die Kammer möglicherweise mehr Vorurteile als das Viermächte-Gremium hat, und daß einige Projekte, die unsere Aufmerksamkeit verdient hätten, das Gremium vielleicht nie erreichen würden.

Vorschlag: ${ }^{166}$ die Kammer der Kunstschaffenden wird ermächtigt, alle Berliner ${ }^{167}$ Filmvorschläge zu prüfen und ihre Empfehlungen an das Viermächte-Zensurgremium zur Genehmigung weiterzuleiten mit der Maßgabe, daß die einzelnen lizenzierten deutschen Filmproduzenten die Möglichkeit haben, das Viermächte-Gremium direkt anzusprechen, wenn sie glauben, daß sie von der Kammer nicht fair behandelt worden sind.

4. Herr Wilder schlägt vor, daß unser Vertreter in dem Viermächte-Zensurgremium nicht unbedingt ein Filmexperte sein muß, er meint vielmehr, daß ein politischer Mann mit gesundem Urteilsvermögen der richtige Vertreter unserer Interessen wäre. Oberstleutnant Thompson ${ }^{168}$ hat die nötigen politischen Voraussetzungen, aber er beherrscht die deutsche Sprache nicht gut genug, um über Filme oder Drehbücher entscheiden zu können.

Vorschlag: Nikolai Nabokov ${ }^{169}$ wird so schnell wie möglich nach seiner Ankunft in Berlin an Entscheidungen in grundsätzlichen Fragen beteiligt, und ihm wird die Aufgabe der Vertretung im Filmzensurgremium anvertraut. ${ }^{170}$

Report: Making German Feature Films Again, Billy Wilder to Davidson Taylor (Chief FIM ICD USFET), 21 August 1945; OMGUS 10/16-2/15.

165 Handschriftliche Randbemerkung von Murphy: „A. OK“.

166 Handschriftliche Randbemerkung von Murphy: „B. OK as amended“.

167 „Berliner“ wurde von Murphy handschriftlich beigefügt.

168 Lt. Col. C. A. Thompson war in der Plans and Directives Branch von ICD USFET beschäftigt.

169 Nikolas Nabokov (1903), russ.-amerikan. Komponist, nach 1945 kurzzeitig bei der amerikanischen Militärregierung in Berlin beschäftigt, später Generalsekretär des Kongresses für kulturelle Freiheit sowie künstlerischer Berater des Senats von Berlin.

${ }^{170}$ Handschriftliche Randbemerkung von Murphy: „OK but coordinate with Lt. Col. Thompson." 